Linköping Studies in Science and Technology.

Dissertation No. 2030

\title{
Data Abstraction and Pattern Identification in Time-series Data
}
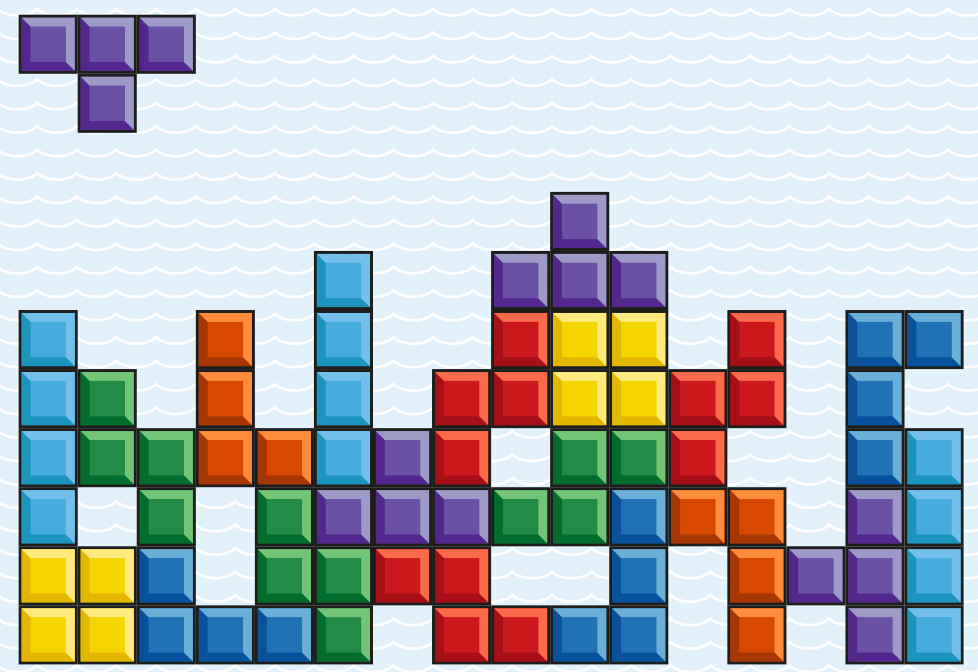

Prithiviraj Muthumanickam 
Linköping Studies in Science and Technology

Dissertation, No. 2030

\section{DATA ABSTRACTION AND PATTERN IDENTIFICATION IN TIME-SERIES DATA}

Prithiviraj Muthumanickam

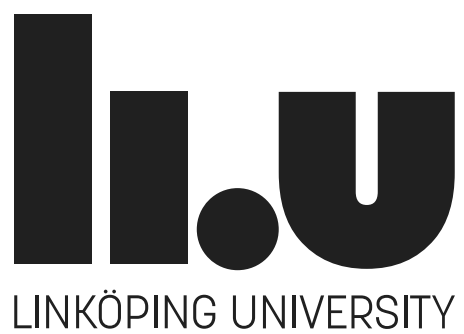

Division of Media and Information Technology

Department of Science and Technology

Linköping University, SE-601 74 Norrköping, Sweden

Norrköping, December 2019 


\section{Data abstraction and pattern identification in time-series data}

Copyright (C) 2019 Prithiviraj Muthumanickam (unless otherwise noted)

Cover image is a representative image of Tetris @\&(C1985-2019. Tetris trade dress are owned by Tetris Holding.

Division of Media and Information Technology

Department of Science and Technology

Campus Norrköping, Linköping University

SE-601 74 Norrköping, Sweden

ISBN: 978-91-7929-965-1

ISSN: 0345-7524

Printed in Sweden by LiU-Tryck, Linköping, 2019 


\section{Acknowledgments}

My sincere thanks to,

Inspirational and motivating supervisors Matthew Cooper, Katerina Vrotsou, Aida Nordman, Jimmy Johansson, Anders Ynnerman for all the guidance, fruitful discussions, advice and support all the way in steering my research career without which I wouldn't be here in this exciting field of research.

Guru Matthew Cooper for educating me and guiding me with your extensive knowledge, perseverance and patience.

Awesome collaborators Lothar Meyer, Jonas Lundberg, Åsa Svensson, Camilla Forsell and Supathida Boonsong for their support.

Exciting colleagues and friends - Lonni, Jouni, Alex, Kahin, Sathish, Rickard, Jochen, Martin, Niklas Rönnberg, Gun-Britt Löfgren for all the good times at the institute.

My loving parents Banu and Muthu for their support, hardships during these years and my wife Sree for illuminating and shadowing me all the way. 



\section{Abstract}

Data sources such as simulations, sensor networks across many application domains generate large volumes of time-series data which exhibit characteristics that evolve over time. Visual data analysis methods can help us in exploring and understanding the underlying patterns present in time-series data but, due to their ever increasing size, the visual data analysis process can become complex. Large data sets can be handled using data abstraction techniques by transforming the raw data into a simpler format while, at the same time, preserving significant features that are important for the user. When dealing with time-series data, abstraction techniques should also take into account the underlying temporal characteristics.

This thesis focuses on different data abstraction and pattern identification methods particularly in the cases of large 1D time-series and 2D spatio-temporal time-series data which exhibit spatio-temporal discontinuity. Based on the dimensionality and characteristics of the data, this thesis proposes a variety of efficient data-adaptive and user-controlled data abstraction methods that transform the raw data into a symbol sequence. The transformation of raw time-series into a symbol sequence can act as input to different sequence analysis methods from data mining and machine learning communities to identify interesting patterns of user behaviour.

In the case of very long duration 1D time-series, locally adaptive and user-controlled data approximation methods were presented to simplify the data, while at the same time retaining the perceptually important features. The simplified data were converted into a symbol sequence and a sketch-based pattern identification was then used to identify patterns in the symbolic data using regular expression based pattern matching. The method was applied to financial time-series and patterns such as head-and-shoulders, double and triple-top patterns were identified using hand drawn sketches in an interactive manner. Through data smoothing, the data approximation step also enables visualization of inherent patterns in the time-series representation while at the same time retaining perceptually important points.

Very long duration 2D spatio-temporal eye tracking data sets that exhibit spatiotemporal discontinuity were transformed into symbolic data using scalable clustering and hierarchical cluster merging processes, each of which can be parallelized. The raw data is transformed into a symbol sequence with each symbol representing a region of interest in the eye gaze data. The identified regions of interest can also be displayed in a Space-Time Cube (STC) that captures both the temporal and contextual information. Through interactive filtering, zooming and geometric transformation, the STC representation along with linked-views enables interactive data exploration. Using different sequence analysis methods, the symbol sequences are analyzed further to identify temporal patterns in the data set. Data collected from air traffic control officers from the domain of Air traffic control were used as application examples to demonstrate the results. 



\section{Populärvetenskaplig Sammanfattning}

Datakällor som simuleringar och sensornätverk i många olika applikationsområden genererar stora volymer av tidsseriedata vilkas egenskaper och karaktäristik utvecklas över tid. Visuella dataanalysmetoder kan underlätta i utforskandet och förståelsen av underliggande relationer och mönster som finns i tidsseriedata, men på grund av dess ständigt ökande storlek blir den visuella dataanalysprocessen komplex. Stora datamängder kan hanteras genom dataabstraktionstekniker som omvandlar rådata till enklare format samtidigt som signifikanta särdrag som är viktiga för användaren bevaras. När tidsseriedata hanteras bör dataabstraktionsmetoder också ta hänsyn till underliggande temporala egenskaperna.

Denna avhandling fokuserar på olika dataabstraktions- och mönsterigenkänningsmetoder, särskilt i fall med stora endimensionella (1D) tidsseriedata och tvådimensionella (2D) spatiotemporala tidsseriedata som uppvisar spatiotemporal diskontinuitet (som exempelvis ögonspårning (eye-tracking)). Denna avhandling föreslår, baserat på dimensionaliteten och egenskaperna hos tidsseriedata, en uppsättning olika dataabstraktionsmetoder för att omvandla rådata till en symbolsekvens. Omvandlingen av tidsserierådata till en symbolsekvens kan fungera som inmatning till olika sekvensanalysmetoder från olika fält som datasökning (data mining) och maskininlärning (machine learning) för att påvisa intressanta mönster av användarbeteende.

I fallet med väldigt långa endimensionella tidsseriedata har lokalt anpassade och användarkontrollerade metoder för uppskattning av data använts för att förenkla tidsseriedata samtidigt som perceptuellt viktiga särdrag bibehållits. Denna förenklade data kan sedan konverteras till en symbolsekvens, och en skissbaserad mönsterigenkänning har sedan använts för att identifiera mönster i symboldata genom mönstermatchning med reguljära uttryck. Viktiga mönster från den finansiella domänen, som skuldra-huvudformationer samt dubbel- och trippeltoppmönster, identifierades med hjälp av handritade skisser på ett interaktivt sätt.

Väldigt långa tvådimensionella spatiotemporala ögonspårningsdata som uppvisar spatiotemporal diskontinuitet omvandlades till symboldata genom skalbar klustrering och hierarkiska klusterfusionsprocesser, som var och en kan parallelliseras. Rådata omvandlas till en symbolsekvens där varje symbol representerar ett område av intresse i ögonspårningsdata. De identifierade områdena av intresse kan också visas i en rums-tid-kub som fångar både temporal och kontextuell information. Genom olika sekvensanalysmetoder analyserades symbolsekvenserna ytterligare för att identifiera temporala mönster i datamängden. Data insamlad från flygtrafikledare i flygledningsområdet användes som applikationsexempel för att demonstrera resultatet. 



\section{Publications}

Paper A: P. K. Muthumanickam, K. Vrotsou, M. Cooper, and J. Johansson. Shape grammar extraction for efficient query-by-sketch pattern matching in long time series. In IEEE Conference on Visual Analytics Science and Technology (VAST), pages 121-130, 2016

Paper B: P. K. Muthumanickam, C. Forsell, K. Vrotsou, J. Johansson, and M. Cooper. Supporting exploration of eye tracking data: Identifying changing behaviour over long durations. BELIV '16, pages 70-77. ACM, 2016

Paper C: P. K. Muthumanickam, K. Vrotsou, A. Nordman, J. Johansson, and M. Cooper. Identification of temporally varying areas of interest in longduration eye-tracking data sets. IEEE Transactions on Visualization and Computer Graphics, 25(1):87-97, 2019

Paper D: P. K. Muthumanickam, A. Nordman, L. Meyer, S. Boonsong, J. Lundberg, and M. Cooper. Analysis of long duration eye-tracking experiments in a remote tower environment. In 13th USA/Europe air traffic management RED seminar, 2019

Paper E: P. K. Muthumanickam, J. Helske, A. Nordman, J. Johansson, and M. Cooper. Comparison of attention behaviour across user sets through automatic identification of common areas of interest. In Proceedings of Hawaii International Conference on System Sciences, HICSS, 2019 



\section{Contents}

Acknowledgments

$\begin{array}{lll}\text { Abstract } & \text { v }\end{array}$

Populärvetenskaplig Sammanfattning vii

$\begin{array}{ll}\text { List of publications } & \text { ix }\end{array}$

1 Introduction 1

1.1 Background 1

1.2 Long duration time-series data sets 2

1.3 Data analysis and visualization 3

1.4 Research challenges 4

1.5 Thesis overview 4

$\begin{array}{lll}2 & \text { Visual analysis challenges } & 7\end{array}$

2.1 Visual effectiveness 8

$\begin{array}{lll}2.2 & \text { Sequence analysis of time-series data } & 12\end{array}$

$\begin{array}{lll}2.3 \text { Computational efficiency } & 14\end{array}$

2.4 Choice of visual analysis methods 14

3 Previous Work $\quad 17$

3.1 1D Time-series data 18

$\begin{array}{lll}3.2 & \text { 2D Spatio-temporal data } & 20\end{array}$

4 Contributions $\quad 27$

$\begin{array}{lll}4.1 & \text { Summary of papers } & 27\end{array}$

$\begin{array}{lll}4.1 .1 & \text { Paper A } & 28\end{array}$

$\begin{array}{lll}\text { 4.1.2 Paper B } & 31\end{array}$

$\begin{array}{lll}\text { 4.1.3 Paper C } & 32\end{array}$

$\begin{array}{lll}\text { 4.1.4 Paper D } & 35\end{array}$

$\begin{array}{lll}\text { 4.1.5 Paper E } & 39\end{array}$

5 Conclusion $\quad 43$

5.1 Data abstraction - Symbolic approximation 43

$\begin{array}{ll}5.2 & \text { Sequence analysis of symbolic data } \\ 5.3 & 45\end{array}$

$\begin{array}{lll}5.3 & \text { Efficient data processing algorithms } & 45\end{array}$

$\begin{array}{lll}5.4 \text { Future work } & 46\end{array}$

$\begin{array}{ll}\text { Bibliography } & 47\end{array}$ 
Publications

Paper A: Shape Grammar Extraction for Efficient Query-by-Sketch

Pattern Matching in Long Time Series

Paper B: Supporting Exploration of Eye Tracking Data: Identifying

Changing Behaviour Over Long Duration

Paper C: Identification of Temporally Varying Areas of Interest in Long-Duration Eye-Tracking Data Sets

Paper D: Analysis of Long Duration Eye-Tracking Experiments in a Remote Tower Environment

Paper E: Comparison of Attention Behaviour Across User Sets through Automatic Identification of Common Areas of Interest 


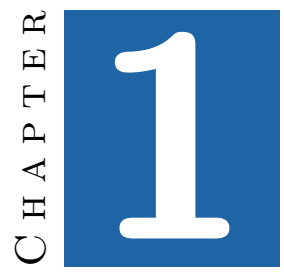

\section{Introduction}

Many application domains generate time-series data sets that are collected across longer time intervals. Visual analysis techniques can aid the process of gaining insights from these data sets and can augment the decision making process of the domain experts. But very large time-series data sets make the visual data analysis process quite complex. Since there is a temporal evolution of the characteristics of the data collected over time, different processing techniques are required for identifying the temporal patterns. This thesis focuses on different techniques for data adaptive and user-controlled data simplification and pattern identification in case of long duration time-series data sets.

\subsection{Background}

In the work of Aigner et. al [3], the authors highlight the importance of making the distinction between the physical dimension of time and the notion of time in information systems. In the latter case the goal is not just to imitate the physical notion of time but to provide tools and techniques through which the underlying patterns in the data and their temporal evolution can be analysed and represented in an intuitive manner through data analysis and visualization.

Whether it is a univariate or multivariate time-series data set, there is a change or evolution in the characteristics of the data such as repeating patterns or anomalies. Hence any analysis method on time-series data should take these characteristics into account because time is not just a numerical parameter among other dimensions of the data [3]. 
While there are many approaches to represent time in information systems, the choice of a particular representation is mainly data and application dependent. Different data characteristics such as abstract or spatial information influence the choice of appropriate visual representations for time-series data. With the increase in the size of the data sets, visualization of the entire raw data becomes complex and hence different data abstraction techniques are required to improve efficiency [1].

In case of very large multi-dimensional time-series data sets with large number of variables, visualization of raw data using techniques such as scatter plots will be difficult and hence reducing the data to lower dimensions such as $2 \mathrm{D}$ or $3 \mathrm{D}$ is one common method available for visualization.

The focus of this thesis is on data analysis and visualization of long duration time-series data, particularly the case of 1D time-series data sets and 2D time-series data sets where spatio-temporal continuity is not guaranteed to exist over time. With the ever increasing size of time-series data, the process of data analysis and visualization becomes quite complex. Hence this thesis investigates,

1. Data adaptive simplification of long duration time-series data using symbolic approximation.

2. Pattern identification in sequential symbolic data using techniques developed for sequence analysis.

\subsection{Long duration time-series data sets}

Some of the examples of long duration time-series data that span multiple time steps and multiple dimensions include, $1 D$ time-series - such as data from sensors, financial domain, simulations, $2 D$ time-series - data such as geographic movement, eye gaze data collected from hours of eye tracking experiments, high-dimensional time-series - data such as activation vectors in the hidden layer of a neural network that changes over multiple epochs.

In the case of $1 \mathrm{D}$ and 2D time-series data sets, the accumulation of data over time can lead to challenges in visualization due to visual clutter and the data analysis process can introduce additional computational complexities. One of the important characteristics of spatio-temporal data is its spatio-temporal continuity over space and time which needs to be taken into account while devising algorithms for data analysis and visualization. For example, object movements such as traffic trajectories exhibit continuity in space and time while in case of eye movement data, there are quick transitions of eye gaze movements to different parts of a scene. Hence, data adaptive algorithms are necessary to understand the temporal evolution of patterns in the data. 
Dimensionality reduction is a common tool to visualize high-dimensional data sets where the data samples from higher dimensions are projected into visually perceivable $2 \mathrm{D}$ or $3 \mathrm{D}$ representations. In case of high-dimensional time-series data, if a dimensionality reduction algorithm is applied independently for each time step, the resulting low dimensional visualization in each time step may not capture the temporal trend in the data leading to temporal incoherence. Hence temporally coherent data visualization methods such as Rauber et. al [100], Jäckle et. al [52] should be taken into account for visual analysis of high-dimensional time-series data.

\subsection{Data analysis and visualization}

Automated data analysis methods can enable identification of patterns over time such as repeating trends, anomalies, location of specific patterns. Pattern recognition algorithms for time-series data sets can be classified into supervised learning methods such as classification, unsupervised learning methods such as clustering and semi-supervised learning methods such as semi-supervised classification, outlier detection. Lin et. al [78] provides a brief discussion of different pattern recognition and data mining techniques for univariate and multivariate time-series data sets. In order to explore the characteristics of time-series data sets, interactive visual data mining approaches combined with automated methods can enable user-centered exploration of data based on the requirements that are significant for the user [117]. Hence, the choice of a particular data analysis method depends on the characteristics of the data and the different tasks that will be performed by a domain user.

The characteristics of the time-series data such as abstract or spatial, dictate the choice of visual representations to be used for visualization. For example, if there is a spatial context associated with time-series data, a space time cube representation could be a candidate for visualization. Based on the nature of user tasks or interaction options, other suitable representations may be necessary even in the case of spatial or abstract time-series data sets. Aigner et. al [3] list three basic questions when choosing a visual representation for time-series data.

1. Data level: What is presented? Different characteristics of data such as quantitative, qualitative, abstract, spatial, event-based, univariate, multivariate dictate the nature of the visual representation.

2. Task level: Why is it presented? Different tasks carried out by the users such as identification and location of temporal patterns, their sequence of occurrence, presence or absence of any anomalies guides us in determining a suitable visual representation. 
3. Presentation level: How is it presented? How do we map time - static or dynamic representations and what factors need to be considered in choosing the dimensionality of the presentation space. An interesting question to answer is whether to use 2D or 3D visual representations [2, 19, 109]. According to Aigner et. al [3] factors such as the analytical goals to be achieved, the application background and user preferences need to be taken into account while choosing between $2 \mathrm{D}$ and $3 \mathrm{D}$ representations. Limiting factors such as occlusions in $3 \mathrm{D}$ visualization can be overcome through additional visual cues, intuitive $3 \mathrm{D}$ interaction techniques and options for data filtering [32].

\subsection{Research challenges}

Very large time-series data sets introduce complexities such as visual clutter, processing time, storage for interactive visual data analysis and exploration. Interactive visual exploration plays a prominent role in analyzing complex data sets because it involves the human in the loop where users perform a hands-on exploration of the data and search for information of interest. With the increasing size of the data, interactive visual exploration becomes difficult. Visualization of 1D time-series graphs and 2D spatio-temporal data sets often become cluttered due to data generated across multiple time steps. Hence the visual identification of interesting short sequences of samples can be very difficult. Data simplification is often necessary for reducing the visual and computational complexity of the time-series data. Researchers have developed a variety of data abstraction approaches that can represent the actual data with a simplified representation while at the same time retaining the essential characteristics of the original data. Since searching for interesting patterns in large time-series data sets can become a computationally heavy problem, different scalable algorithms that are computationally efficient to the increasing volumes of data sets are explored by different methods. Hence the following research goals are laid out in this thesis,

1. Simplification of raw data by transforming the raw data samples into a simple format while at the same time preserving significant features that are important for the user.

2. Sequence analysis of simplified data to identify patterns of interest for a user.

3. Efficient data processing algorithms to improve the computational complexity when dealing with very large time-series data sets.

\subsection{Thesis overview}

This thesis is divided into three parts 1: Chapters 2 and 3 discuss the visual analysis challenges in working with large data sets and the related work, 2: Chapters 4 and 
5 summarize the aim, method description, results and conclusion of the included publications, and 3: the included publications.

Chapter 2 describes the challenges such as visual effectiveness and computational efficiency that need to be addressed when dealing with large time-series data sets. Specifically, the need for data abstraction and simplification techniques that can convert raw data into simplified representation which can be used as input to sequence analysis methods are discussed.

Chapter 3 summarizes the related work specifically in the case of symbolic approximation and sequence analysis in case of long 1D time-series data and long duration 2D spatio-temporal eye tracking data sets. Data simplification into symbolic approximation enables the use of different sequence analysis approaches such as grammar based analysis for pattern identification, Hidden Markov Models and Sequence mining.

Chapter 4 presents the contribution of this thesis. For every included publication in the thesis, an overview of the aim, methods and results is provided.

Chapter 5 provides a description concerning the nature of very large time-series data sets and how the included publications address the challenges that were outlined in the earlier Chapters 2 and 3. Also, the chapter proposes possible directions for future work. 



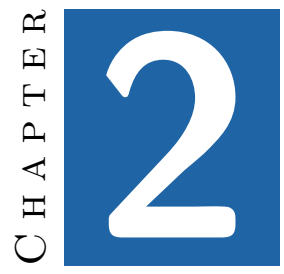

\section{Visual analysis challenges}

Visualization of the entire raw time-series data is usually not feasible and rarely reveals any further insights for the domain user. Large time-series data sets can also hinder the effectiveness of exploratory data analysis [9] and user interaction where the advantage of having the domain user in the loop suffers a setback.

The well-known information seeking mantra introduced by Ben Shneiderman in 2003 [109] defines exploratory data analysis as a three step process — 'overview first, zoom and filter, then details-on-demand'. In order to deal with complexity of large volumes of data, a scalable visual analysis mantra was later proposed by Keim et.al [58] — 'analyse first - show the important - zoom, filter and analyse further - details on demand'. Hence, in order to deal with large volumes of data, data analysis methods can be used as a first step to simplify the data by grouping related data items, identify interesting parts of data for further investigation by an analyst or perform detailed analysis using drill down operations.

According to Guo et.al [42] visual analysis techniques face two kinds of challenges from large data sets: Visual effectiveness and Computational efficiency. Visual effectiveness can be improved by converting the raw data into a simplified representation, while the computational complexity of dealing with large data sets can be handled using, for example, scalable algorithms. In case of time-series data sets, the raw data can be converted into a simplified representation by taking into account the temporal characteristics of the data and different classes of sequential data analysis techniques can be applied to the time-series data to find meaningful patterns. 


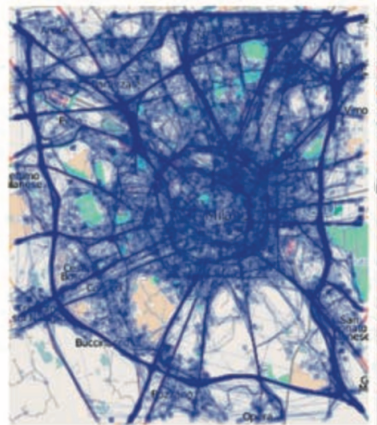

(a)

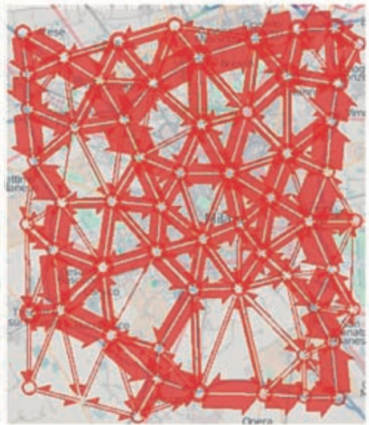

(b)

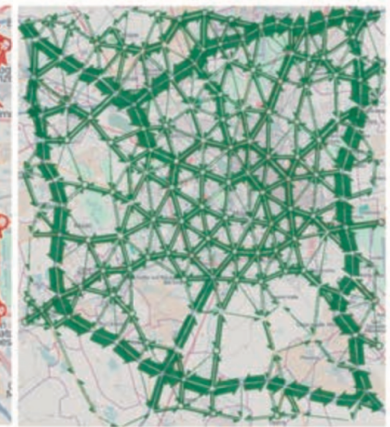

(c)

Figure 2.1: Improving visual effectiveness through spatial aggregation of car trajectory data. (a) Raw data drawn with 10 percent opacity (b) Trajectories after aggregation (c) Lower degree of abstraction. Image courtesy of Andrienko et. al (C2010 IEEE.

\section{$2.1 \quad$ Visual effectiveness}

A large data set can lead to overlapping of data items and the resulting visual clutter makes it very hard to perceive patterns in the data set [60]. For example, in the case of large time-series data sets, displaying the entire raw data set on a commodity screen with limited resolution would create visual clutter and can hinder users from gaining any meaningful information. Hence, efficient data abstraction techniques are required to reduce the complexity of the data set and increase visual effectiveness for identifying meaningful patterns. Furthermore, as the characteristics of time-series data sets evolve over time, an appropriate choice of visual representations is necessary to identify temporal change of the patterns.

\section{Data abstraction}

Data abstraction can transform the raw data samples into a simple format while at the same time preserving significant features that are important for the user $[31,110$, 118]. Large data sets containing billions of raw data samples can be pre-processed using standard data abstraction techniques and can be converted into a simple format.

Sampling is one common technique for data abstraction which determines a representative subset of the original data while maintaining its essential characteristics. Specialized sampling methods such as Visualization-aware sampling (VAS) [94] maximise visual fidelity to produce high quality visualizations. Querying is another form of data abstraction where a fixed subset of the data is determined a-priori for further data processing [26, 27]. A combination of different data abstraction methods such as sampling or binned aggregation and interactive querying was 


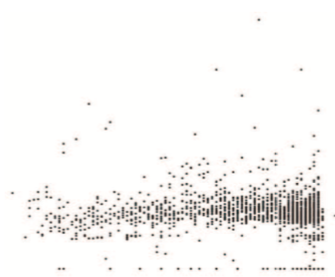

(a)

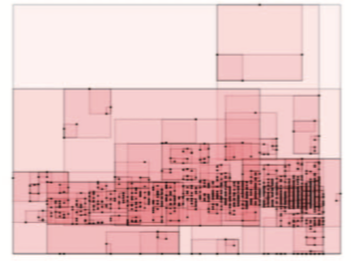

(b)

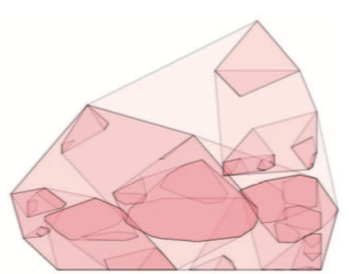

(c)

Figure 2.2: Improving visual effectiveness through Hierarchical aggregation. (a) 2D Scatter plot visualization (b) Aggregation using bounding box (c) Aggregation using convex hull. Image courtesy of Elmqvist et. al (C2009 IEEE.

proposed in imMens [80], an interactive, web-based WebGL system. Such a hybrid methodology allows for both data simplification and interactive scalability for real time interaction along with scalable visual summaries of the data set.

Oliveira et. al [26] highlight some prominent data abstraction methods such as dimensionality reduction [115], data sampling [94], segmentation, cluster analysis [53], density mapping [55], shifting of data points [61] and data aggregation [31] with drill-down capabilities to visualize subsets rather than the entire data set $[41,120]$. While there are many data abstraction techniques, the choice of a particular data abstraction technique is data, user and task dependant. For example, in case of raw trajectory data shown in Figure 2.1(a), different degrees of spatial abstraction better reveals the car mobility pattern as shown in Figure 2.1(b) and (c). In case of a $2 \mathrm{D}$ scatter plot visualization as shown in Figure 2.2, different hierarchical aggregation approaches can be used to approximately represent the dense data samples using bounding boxes or data adaptive convex hulls in a hierarchical manner.

In general, Aigner et. al [2] mention two principal approaches to data abstraction: Aggregation, when data values are aggregated in part or as a whole and Featurebased, by visualizing parts of the data that satisfy a user-defined criterion.

General requirements for data abstraction methods. While the choice of an appropriate data abstraction method is application dependant, the following general requirements $[34,110]$ are necessary for any data abstraction algorithm that computes approximate representations of large data sets,

1. the approximate representation of data must be accurate.

2. Computations must be carried out in main memory to avoid disk I/O overhead.

3. Algorithms must be computationally efficient.

Classification of data abstraction methods. According to Cui et al. [24] abstraction techniques can be classified into two groups. Namely, abstraction 


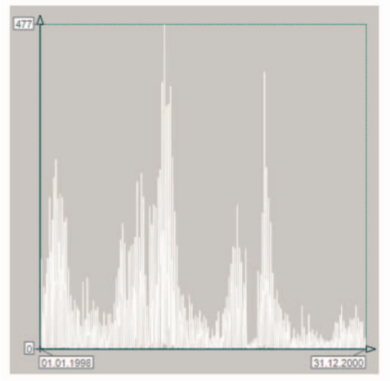

(a)

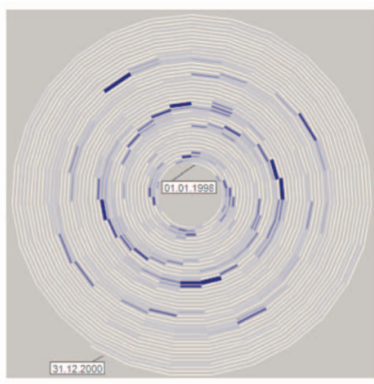

(b)

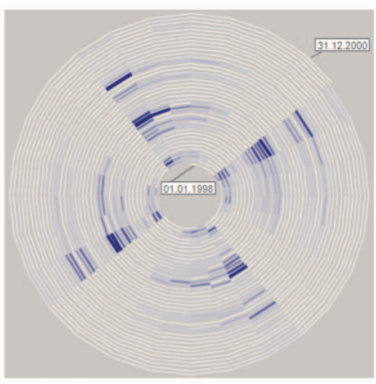

(c)

Figure 2.3: Different visual representations of time-series data set representing number of influenza cases over a period of three years. (a) Time-series plot (b) SpiralGraph encoding 27 days per cycle (c) SpiralGraph encoding 28 days per cycle (Clearly portrays the periodic pattern in the data over time). Image courtesy of Aigner et. al (C2007 IEEE.

in data space and abstraction in visual space. Techniques such as clustering, segmentation, projection and dimensionality reduction fall under the category of abstraction in data space. Keim et.al [59] list different visual space abstraction methods such as interactive filtering, zooming, distortion, linking and brushing. While data space abstraction reduces the size of data and at the same time retaining its characteristics, visual space abstraction enables clutter reduction by assigning more screen space to interesting data elements than to less interesting elements.

Data abstraction for time-series data. Even though there are many data abstraction approaches available, they cannot be applied directly to time-series data as they have temporal ordering and relationships between data samples over time. For example, streaming data sets containing incoming data samples over time require different dynamic processing methods for data analysis and visualization. In the work of Andrienko et. al [10], incremental clustering is performed on spatiotemporal events and their evolution over time is visualized and analyzed for event stream monitoring. In the work of Shurkhovetskyy et.al [110], the authors provide a thorough review of different abstraction methods that are available for visualizing large time-series data from the perspective of visualization and visual analysis. While there are many methods available for data abstraction, the choice of an appropriate method is application specific and depends on the characteristics of the data, the users and the user's task.

\section{Visualization}

Time-series data sets require visualization techniques that are suited for displaying the characteristics of data that evolve over time. The appropriate choice of a 


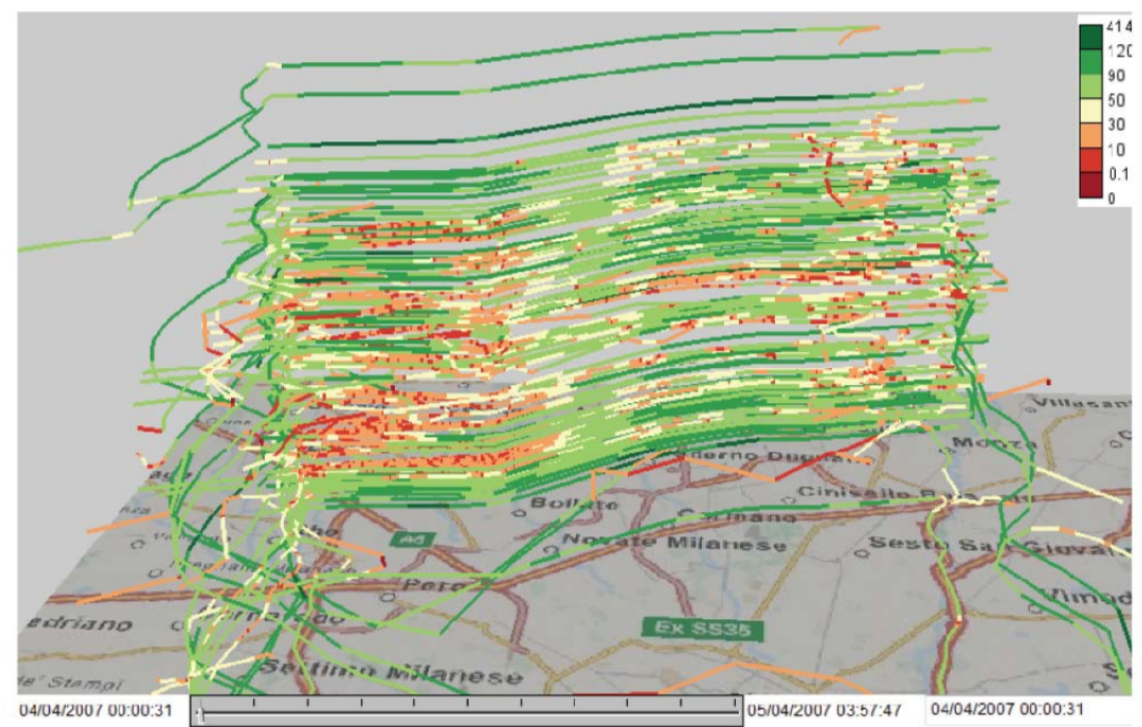

Figure 2.4: Space-time cube visualization of the trajectories of vehicle movement along with contextual information. Colors represent the distribution of speed values in space and time. Image courtesy of Andrienko et. al (C2013 IEEE.

visualization technique can improve the visual effectiveness in conveying temporal patterns in the data samples. For example, a time-series data set representing the total number of influenza cases over a period of three years is displayed in Figure 2.3 using different visualization techniques. Among the three visual representations (a)-(c), the spiral graph plot in Figure 2.3 (c) most clearly portrays the periodic patterns in the data set when compared with other representations.

When there is a spatio-temporal context associated with the data set, space-time cube representations [11] as shown in Figure 2.4 are also suitable for displaying the temporal characteristics of the data. Small multiples, animations, connected scatter plots, parallel coordinates [54] are suitable for visualizing multi-variate data over multiple time steps. An extensive survey of different visualization techniques for time-series data can be found in the work of Aigner et al. 2011 [3] and interactive online versions can be found at http://survey.timeviz.net.

The choice of an appropriate visual representation for the time-series data set can also be made user-centric. Based on the interests of the user's task, user-centric visualization framework such as the one proposed by Tominski [114] for event data - 'Event-based visualization' can be used. In this framework, for search related tasks, the users can specify their search interests as event types and the visual representations are adjusted automatically to match the detected events. This 


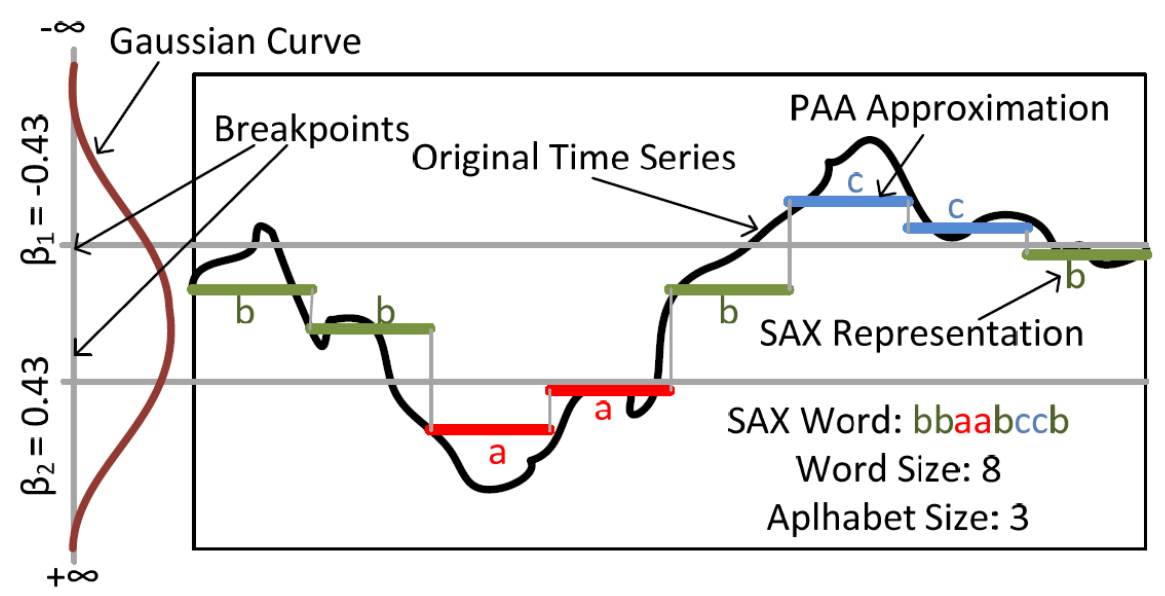

Figure 2.5: The raw time-series data is transformed into the symbol sequence bbaabccb using Symbolic aggregate approximation (SAX) [75]. Image courtesy of Alam et. al (C2013 IEEE.

approach allows the generation of representations that are suitable for a particular search task of a user.

\subsection{Sequence analysis of time-series data}

Sequence analysis is a class of methods that can enable identification of patterns in a time-series data set. They can be applied either to the raw time-series data or to an intermediate simplified representation in order to reveal repeating patterns, anomalies and patterns of interest specified by a domain user using input queries. Since dealing with very large raw time-series data can hinder visual effectiveness and increase computational complexity, the data abstraction methods that were discussed earlier can convert the raw time-series data into an intermediate representation, for example symbolic representation, that can then act as input to sequence analysis algorithms. For example, transformation of raw time series data into a symbol sequence using Symbolic Aggregate Approximation (SAX) $[4,75]$ is portrayed in Figure 2.5.

Sequence analysis methods. Once the raw time-series data is converted into a symbol sequence, identification of repeating patterns and anomalies [107] can be performed through grammar-based approaches such as Sequitur [89]. Since they are greedy grammar-based methods, finding all possible patterns using a smallest complete grammar is an NP-hard problem [74]. Hence, a domain user driven sequence analysis of symbolic data enables an analyst to drive the data analysis 

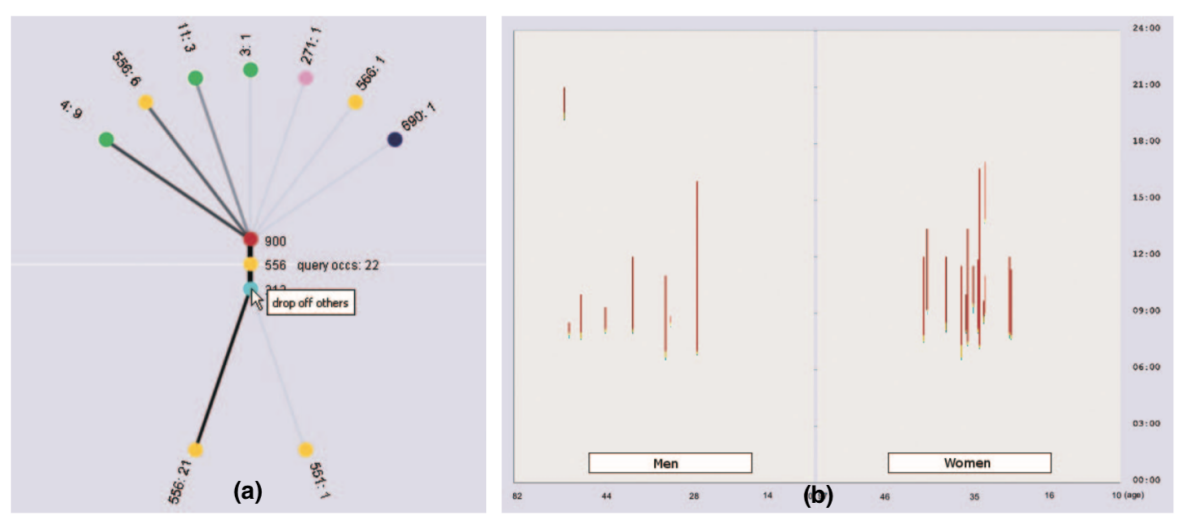

Figure 2.6: User-driven sequence analysis of event-sequences. (a) ActiviTree interface with a selected query sequence: drop off others $->$ travel by car $->$ work (b) Temporal distribution of the explored query sequences across two sets of users conveying the pattern that the query sequence is mostly performed by women. Image courtesy of Vrotsou et. al (2009 IEEE.

and exploration process to find patterns based on their own interest. Methods such as ActiviTree [117] as shown in Figure 2.6 allow the exploration of symbolic data by selecting a symbol of interest and performing a sequence exploration by growing a tree. Since the entire analysis process is user driven, a user can search for patterns such as frequently occurring sequence paths as well as less frequent sequences. Pattern-growth based sequence mining [95] is a family of algorithms that addresses both the concerns of effectiveness (mining sequential patterns interesting to the user) and efficiency (focusing the search where interesting patterns can exist) of mining patterns. While on one hand, this strategy allows the user to push deeper constraints [96] during the mining process thereby leading to more effective pattern detection process and on the other hand, a divide-and-conquer strategy is used. In this process, the search space is divided into smaller sub-sets, that are in turn recursively searched for patterns and hence allows for more efficient search of patterns in the data.

Another approach for sequential data analysis is a Hidden markov model (HMM) [13] that finds a probabilistic description of sequential patterns in symbolic data, both for smoothing noisy high-frequency data and for predicting missing observations (e.g. due to sensor malfunction). They are used for clustering of sequential or time-series data [93]. 


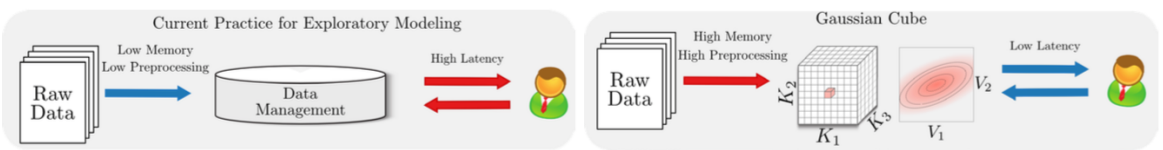

Figure 2.7: Low-latency exploratory modeling using Gaussian cubes. Image courtesy of Wang et. al (C2017 IEEE

\subsection{Computational efficiency}

Large amounts of data in their raw format can increase the computational complexity and hence lead to poor responsiveness of the overall system [29, 35]. Computational complexity can be overcome in different ways such as design of scalable algorithms [7], usage of advanced data structures [79, 119], advanced memory management techniques for retaining data in memory, disks [58].

For example, a parallel rendering architecture can aid the visual analysis process by providing efficient visualization and real-time interaction for a domain user. A parallel rendering architecture was proposed by Piringer et.al [97] that synchronizes and communicates between a main application thread and a visualization thread along with an architecture for generating incremental previews of visualization. This system provides rich visual feedback at interactive rates for very large data sets of the order of gigabytes and ensures responsiveness during exploratory data analysis.

When the size of the data sets does not fully fit in the main memory, parallelized out-of-core computation methods are necessary for analyzing the data set. For example, for analyzing very large traffic movement data sets, a scalable event clustering method was proposed by Andrienko et. al [7] where the data can be processed without simultaneously loading all of the data in the main memory.

Nanocubes [79] and Gaussian cubes [119] are used for efficient model fitting, data management and exploratory data analysis. As shown in Figure 2.7, building of data models to explore a data set through repeated scans of data can increase the latency for user interaction while the construction of data cubes such as Gaussian cubes can support low-latency exploratory modeling through computation of model parameters in real-time. Hence, these methods enable interactive, query-based visual analysis for very large spatio-temporal data sets, where a hierarchy of data aggregation is pre-computed for efficient user interaction and visualization.

\subsection{Choice of visual analysis methods}

In order to gain meaningful information from the data set, an effective visual analysis process should provide effective visual representation, efficient human interaction for analysis of patterns in the data and should be computationally 
efficient and scalable to process very large data sets. According to the design triangle framework of Miksch et.al [82], the characteristics of the data, users and user's task should be taken into account when choosing an appropriate method for visualization and data analysis. They provide a design framework with three sets of questions that need to be answered when choosing suitable visual, analytical and interaction methods,

1. Data: What is the nature of the data set?

2. Users: Who is going to use the system?

3. Tasks: What are the tasks of the users?

Nature of data. Time-series data has multiple characteristics such as time primitives (instant, interval, span), scale (quantitative, qualitative), frame of reference (abstract, spatial), kind of data (events, states), dimension (uni-variate, multivariate), temporal arrangement (linear, cyclic, branching), availability (stationary, streaming data) [1, 2, 3, 82]. For example, in the case of large temporal multidimensional data sets, when projecting the data samples from different time stamps to lower dimensions, the temporal ordering between data samples needs to be taken into account so that the problem of temporal-incoherence can be avoided. Methods such as temporal MDS plots [52] and dynamic t-SNE [100] take the temporal incoherence factor into account while projecting high-dimensional time-series data to lower dimensions. The presence of noise in a real-world setting needs to be taken into account which affects the applicability of data abstraction and visual analysis [57]. Hence, different characteristics of time-series data sets need to be taken into account while designing effective visual analysis methods.

Users. User interests specific to their application domain should be taken into account to make an appropriate selection where the data characteristics and user requirements can dictate the choice of a visual analysis method.

User Tasks. The nature of the user's tasks should also be taken into account so that valuable information is not lost in the simplification process. The work of Aigner et. al [3] lists different user tasks such as classification, clustering, search and retrieval, pattern discovery, anomaly detection and other sequence analysis tasks. In the work of Shurkhovetskyy et.al [110], they propose additional considerations when choosing an abstraction method for time-series data analysis such as indexing data structures like R-tree to speed up data access and partitioning of the data for suitable level of detail representation. 



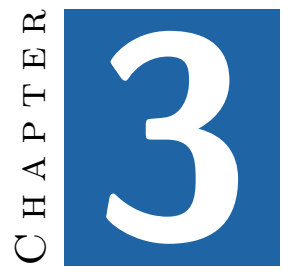

\section{Previous Work}

As discussed in the previous chapter, data abstraction can be used to improve visual effectiveness and data analysis of very large time-series data sets. This thesis focuses particularly on data abstraction and analysis of very long duration 1D time-series data and 2D spatio-temporal data sets exhibiting spatio-temporal discontinuity. For example, spatio-temporal geographic movement data such as human or animal migration exhibit data continuity in space and time due to the nature of the movements of objects. But in the case of spatio-temporal eye movement data sets, they are governed by inertia with quick transition of eye gaze movements to different parts of a scene and hence they lack the spatio-temporal continuity found in geographic movement data sets. Hence, this characteristic of spatio-temporal discontinuity in time-series requires different analysis methods than those that are available for movement analysis.

This thesis focuses specifically on data abstraction into symbolic representation that takes into account the temporal characteristics of the data. Transforming the raw data into a symbol sequence enables application of sequence analysis tools such as those found in text processing and bio-informatics communities [72] to analyze repeating patterns, anomalies, user query based pattern identification. Also, using symbolic approximation, the original data can also be transformed into smaller or less complex symbol components that are beneficial for storage or computation [112]. This chapter subdivides the discussion of related work into (1) Data abstraction based on symbolic approximation of temporal data and (2) Sequence analysis of symbolic data to uncover patterns of interest. 


\subsection{D Time-series data}

Time-series data mining is a vast field with numerous methods focused towards motif discovery, anomaly detection, clustering, classification, segmentation, prediction and query-by-content. A thorough review of different techniques can be found in $[33,36,37,78]$. While there are many high level representations that are available for time-series data analysis, this thesis focuses specifically on symbolic representation and approximation that can avail the enormous wealth of algorithms from text processing and bio-informatics communities such as Markov models, Suffix trees, Decision trees, Hashing [77].

\section{Data abstraction - Symbolic approximation}

The advantages of transforming raw time-series data into symbol sequence such as identification of patterns using sequence analysis methods, efficient data storage and reduced computational complexity is highlighted in Lin et. al [77]. As a first step, methods such as Symbolic aggregate approximation (SAX) [75] transforms the timeseries data into a symbol sequence. SAX simplifies the time-series by segmenting the data based on user specified segment length and assigns a symbol to each segment. But this approximation leads to smoothing of perceptually important points (PIP) as shown in Figure 3.1. Multiple methods have been proposed to address this limitation by storing additional information with each symbol such as information on slope, maximum and minimum of amplitude points [85]. A brief state of the art report on different methods that are available for symbolic approximation of time-series data is available in [25]. Shurkhovetskyy et. al [110] proposes a detailed classification of different data abstraction methods for time-series data.

\section{Sequential data analysis of symbolic data}

Data analysis of the symbol sequence of 1D time-series enables identification of patterns that are of interest to an analyst.

Grammar-based motif identification. Since the raw data is labelled into symbol space, grammar based approaches such as Sequitur [89] can be used to identify repeating patterns and anomalies in the data set. Finding the list of all possible patterns using a smallest complete grammar is an NP-hard problem [74] and hence Sequitur [89] and other methods based on it are greedy-grammar based approaches.

Query-by-content based motif identification. Query-by-content based approaches involve the domain user in the loop where they can specify motifs using different input mechanisms. The analyst can specify the query by selecting a part of the input raw data using time boxes and this selected pattern is then searched for across the whole data set $[47,48]$. This query specification model was made flexible 


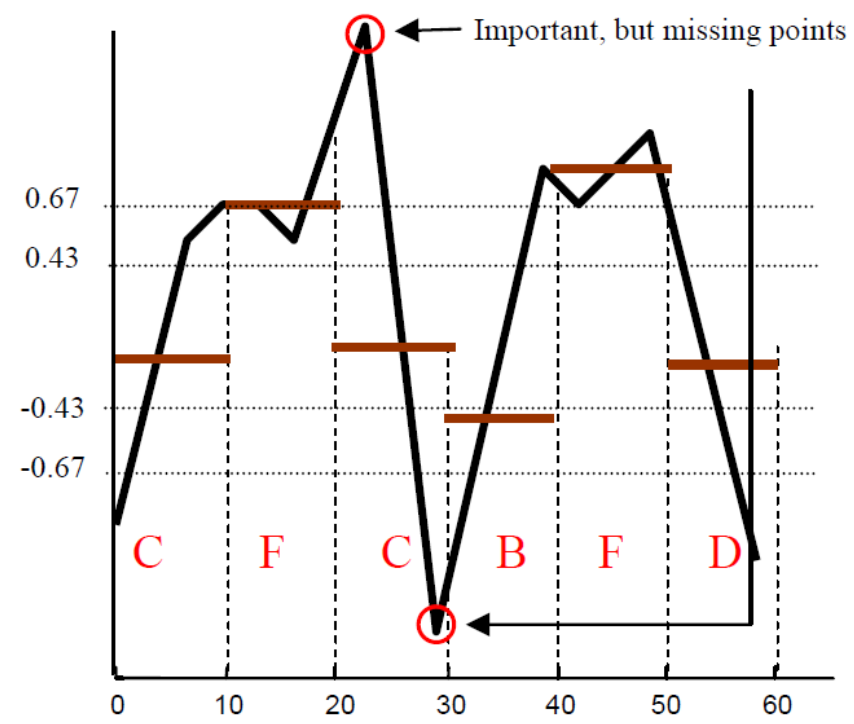

Figure 3.1: Raw time-series data is transformed into the symbol sequence CFCBFD using Symbolic aggregate approximation (SAX) [75]. Perceptually important points (shown in red circles) are not captured in the symbolic approximation. Image courtesy of Lkhagva et. al (C2006 IEEE.

using approaches such as variable timeboxes [62], angular queries and slopes [49], query adjustment functions [17], pre-defined shape templates [16], Querylines [102], relaxed selection technique [51]. One commonality among these approaches is for the user to pan through the data to find patterns of interest, sketch an approximate pattern over them and then search for similar patterns across the entire data. This process may become tiresome if the time-series data is long. When a domain expert knows the pattern to look for, it is common to manually sketch an approximate pattern and search for similar patterns in the time-series data [121]. Most common shapes such as spikes, sinks, plateau, valley are computed as templates and a domain user can select a template and search for similar matches in the time-series data [39]. All these approaches utilize the knowledge of domain experts to search for patterns of interest.

\section{Visualization of time-series data}

After simplification of time-series data into a symbol sequence, the patterns in the data can be visualized using sub-sequence trees such as VizTree representation [76]. 


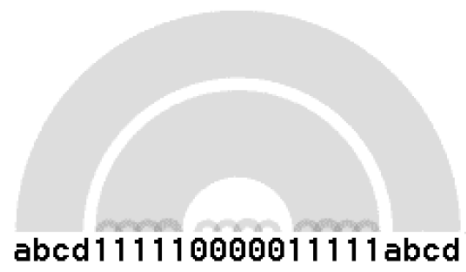

(a)

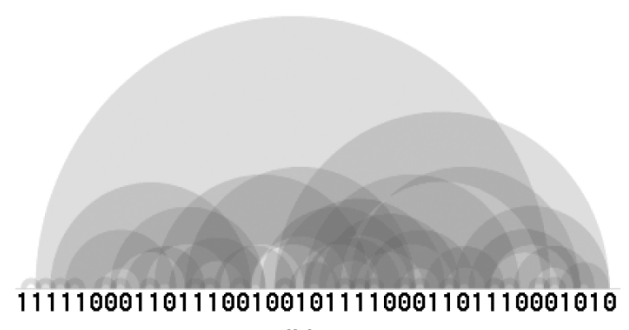

(b)

Figure 3.2: Visual representation of repeating patterns in symbol sequence using Arc Diagrams. Visual clutter due to small and repeating sub-sequences are visible in (b). Image courtesy of Wattenberg et. al (C2002 IEEE.

Colors and other visual attributes of the tree can be used to represent the frequency and other properties of patterns.

Arc Diagram [122] representation allows identification of repeated patterns in the symbol sequence through translucent arcs between repeated sub-strings. But this approach can lead to visual clutter when there are many small repeated sub-sequences as shown in Figure 3.2. Chaos game inspired bitmaps are used to represent the symbol sequence as thumbnail images [66]. One unique feature is that the representation can also allow for calculation of distance between two time-series providing efficient computation. Hence it also allows for classification, clustering, and anomaly detection based on time-series thumbnails.

Coloured rectangles [45] are used to represent frequently occurring patterns in the time-series using rectangles of different sizes. They are used to highlight the occurrences and hierarchical relationships of patterns in the time-series data. Sector visualization [73] based approach is composed of several sectors where each one represents a sub-string pattern of the symbol sequence. Different visual parameters of the representation such as color of the sector, radius are used to map the symbol of a pattern and its length.

\subsection{D Spatio-temporal data}

Various application domains such as human-computer interaction, media and marketing, simulation studies employ eye tracking sensors to collect eye gaze samples which can be analysed to understand the user behaviour. Eye tracking data is a collection of two dimensional eye gaze points over time. When a user is looking at a particular region of a stimulus, raw eye gaze points are aggregated around that region and we call them fixations, while rapid eye movements between fixations are called a saccade $[14,50,104]$. Figure 3.3 portrays the 2 D representation 


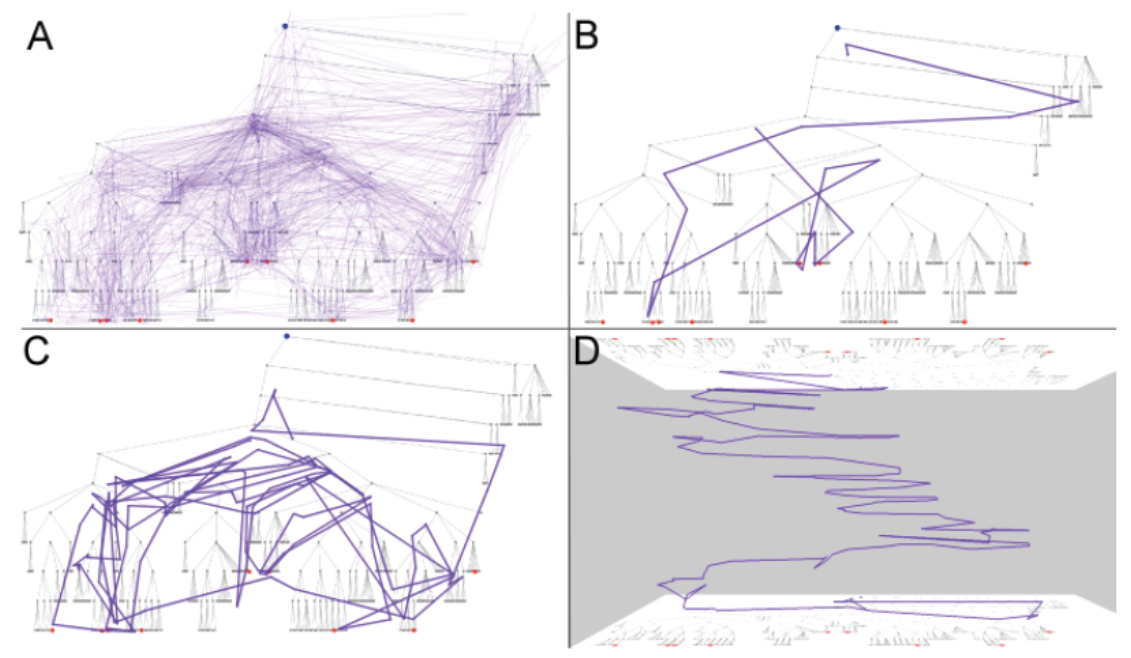

Figure 3.3: Visualization of multiple eye gaze trajectories using $2 \mathrm{D}$ and $3 \mathrm{D}$ visualization. (a) Multiple trajectories are overlaid with the context with a $20 \%$ opacity. (b) and (c) Individual eye gaze trajectories. (d) A single eye gaze trajectory visualized in a Space-time cube. Image courtesy of Andrienko et. al (C2012 IEEE.

and 3D Space-time cube visualization of multiple eye gaze trajectories. Due to the increase in the sampling rate of eye tracking sensors and with long duration eye tracking experiments, very large eye gaze data sets are generated across multiple users and user trials which makes their visualization very cluttered. While many quantitative metrics are available to analyze the eye gaze samples, qualitative analysis of very large eye tracking data sets becomes computationally complex.

\section{Data abstraction - Symbolic approximation}

Data abstraction of eye tracking data involves transformation of raw eye gaze samples into symbol sequence which can be analyzed using sequence analysis methods to identify patterns in eye gaze behaviour. Eye gaze samples can be labelled based on their spatio-temporal characteristics into a symbol sequence. Based on the the time span between successive eye gaze samples, they can be labelled predominantly into fixations (periods of long attention duration) and saccades (quick successive eye movements). Fixation clusters can be labelled further into Areas of Interest (AoI) based on their spatial proximity. Different methods are available to label eye gaze samples and we can classify them into two predominant groups: Image analysis based methods for labelling eye gaze 
data and Labelling the data based on spatial characteristics (AoI) using clustering algorithms.

Image analysis based data abstraction. Eye gaze samples are labelled by taking into account the distinct objects in the scene that are monitored by the user using image processing algorithms [98]. When labelling distinct objects in the scene, first a manual annotation is performed on the distinct objects and the annotated objects are then automatically identified [92]. Kurzhals et al. [70, 71] perform spectral clustering on image thumbnails across different video recordings. Thumbnails of size $100 \times 100$ pixels around each eye gaze point are extracted from multiple video recordings and clustered accordingly. Automatic annotation using scale-invariant feature transform were also used to detect distinct features in videos [64]. Numerous other methods of labelling eye gaze samples based on the underlying context are discussed in $[70,71,92,98]$. While computing the distinct object labels in the scene over time, their associated spatial context is lost and hence, labelling the eye gaze data samples using spatio-temporal characteristics are also necessary.

Spatial context based data abstraction. Labelling of eye gaze samples based on distinct objects in the scene can facilitate the understanding of what are the objects in the scene that gained the attention of the user. While labelling the samples based on their spatio-temporal characteristics aims to reveal how a user inspects and monitors a scene that contains distinct objects and how do the eye gaze movements evolve over time. Another important distinction between the two approaches is that the data resolution of object based labelling will be less when compared with spatio-temporal labelling. For example if an object is moving in the scene and the visual attention of the user is on this object, the eye gaze points will be labelled with the object id, while spatial labelling leads to different labels based on the nature of the object's trajectory. Based on the answers that one would like to gain from the eye tracking experiment, an object id can also be associated to spatial labels over time.

A common approach to spatio-temporal labelling is to subdivide the scene using a grid based approach with a predefined grid resolution and assign appropriate labels of the grid cells to eye fixation points that fall within them. The predefined resolution of the grid determines the accuracy and the number of identified AoIs [18, 38]. The disadvantage of a predefined grid based approach is, they are not dependant on the characteristics of the data and when we are dealing with long-duration experiments, they are not flexible with the temporal evolution of a user's attention over time. Over et. al. [91] constructed Voronoi cells around eye gaze fixation points based on the fixation density, so that areas of high fixation density lead to small Voronoi cells and vice-versa. Example of a Voronoi based spatial tessellation and aggregation of eye gaze points is shown in Figure 3.4. Simple binning [23] or percentile mapping [65] can also be applied to fixations for labelling the eye gaze data. However, these methods omit the temporal properties while 


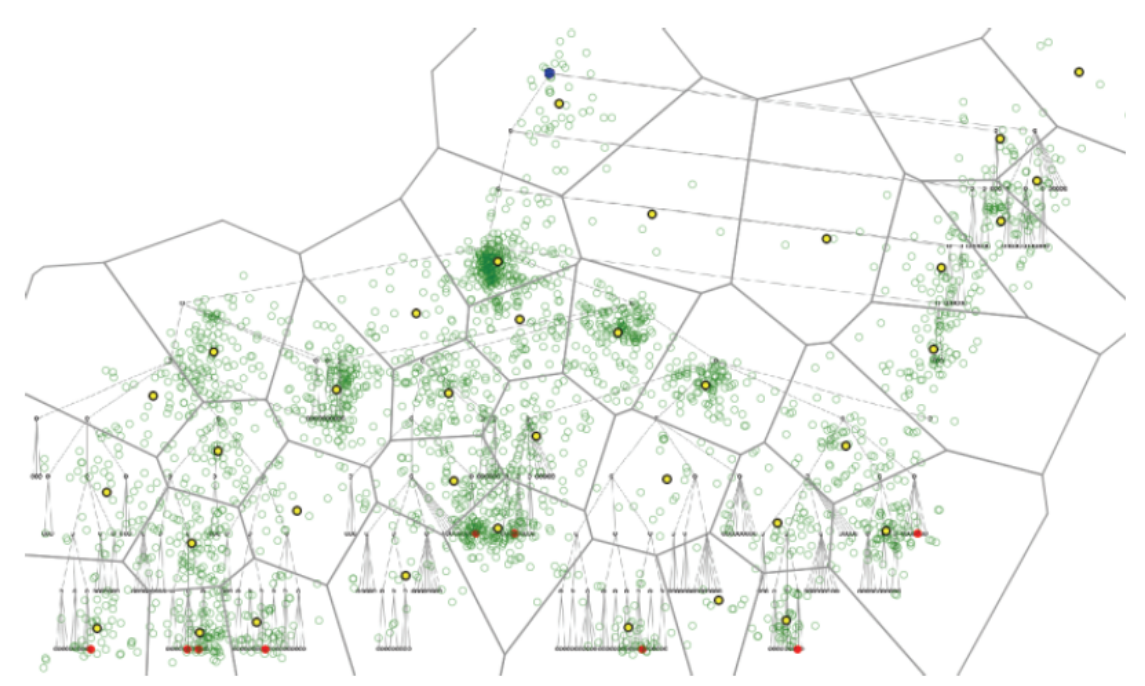

Figure 3.4: Aggregation of eye gaze trajectory using Voronoi based spatial tessellation. Image courtesy of Andrienko et. al (C2012 IEEE.

labelling the data samples, due to data aggregation over time.

For the analysis and visualization of eye tracking data, different approaches from the area of Geographic Information Science (GIS) are proposed in the work of Andrienko et al. [6]. Their work provides a thorough overview of visual analysis approaches with their merits and drawbacks for analyzing eye movement data. Unlike the trajectories of moving objects, eye gaze data is not spatially continuous over time, since eye movements involve frequent attention switching over the scene. In case of long-duration eye-tracking recordings, this becomes even more complex. Hence the spatio-temporal clustering methods from GIS are not suitable for direct application to long-duration eye tracking experiments.

Many data clustering approaches such as Mean shift [40, 103] and Gaussian Mixture Models $[83,105]$ have been used to identify clusters from fixation points, but even applying these methods to long duration eye tracking data sets leads to data saturation with very few labels or spatially coherent clusters across different time intervals get different labels. Therefore, these methods are not suitable for direct application on long-duration data. While the curse of multi-dimensional data is its sparsity in higher dimensions, eye tracking data is faced with high accumulation of data samples over time which can distort the local information. In general, for very long eye tracking data sets, identification and labelling based on spatio-temporal characteristics becomes complex due to data saturation over time. 


\section{Sequential data analysis of symbolic data}

Once the raw eye gaze data is transformed into an Area of Interest label sequence, different sequence analysis methods from data mining or machine learning can be employed to identify interesting patterns of user behaviour.

Transitions between the different areas of interest labels can be modelled as transition matrices and if the matrix is dense with most of the cells containing transition information, it indicates an extensive search on a display while sparse matrices indicate more efficient and directed search. But such matrix representations do not convey the temporal aspects of the visual scanning behaviour. Transitions between different labels can also be modelled as first order Markov chains [101] where the transition to a future area of interest is dependent only on the present area of interest. The Shannon entropy coefficient of the Markov model is then computed to quantify the transition across areas of interest.

While the transition matrices and Markov models estimate the conditional probability of the sequence to the first order i.e, only between two AoIs, thereby looking at only one step of the sequence, while higher order matrices suffer from less data (transitions) to calculate an accurate estimate. Reinforcement learning algorithms learn to predict future data sequence based on past information [46]. Once an eye gaze transition happens from one AoI to another, instead of simply updating the transition probability between them, the method associates the first and second AoI and all expected subsequent AoIs based on prior visits to the second.

When an eye tracking experiment is conducted across multiple users performing the same scenario, sequence analysis can provide insights into common and explicit patterns across different users. In order to analyze symbol sequences across multiple users, similarity metric based algorithms from bio-informatics, such as Levenshtein distance [90] are used to compute the distance between symbol sequences of different users $[30,98]$. A similarity score is computed based on the minimum number of string insertions, deletions, and substitutions required to convert from one string into another. Anderson et. al [5] provides a state of the art report on different scan path comparison methods.

Hidden Markov models are applied to AoI labels and the hidden states that drive the sequence of AoIs are computed in $[22,56]$. They are robust to noise but have difficulty in identifying the model parameters and the number of hidden states that make up the model. The sequence of AoI labels can be seen as a sequence of events, where each event corresponds to a visit to an AoI. This makes it possible to use sequence mining techniques such as Eloquence [116] and other sequence mining approaches that enables user driven investigation of the user's visual scanning strategies during an experiment. Sequential patterns in eye movements which may be shifted by a time span, can be easily detected based on their frequency of occurrence in the data set. 


\section{Visualization of eye tracking data}

After data simplification, numerous visualization techniques are available to visualize eye tracking data sets. The Space-Time Cube (STC) [11] provides the spatio-temporal context and can be used to display the time evolving nature of areas of interest in the visual attention along with the underlying scene information $[28,67]$. Multiple methods have used a combination of coordinated views to overcome the problem of visual clutter in STC displays. For example, synchronized scarf plots and timeline views are used in ISeeCube [69]. Among alternative visualizations that try to reduce visual clutter, scarf plots are an efficient way of representing AoIs and their evolution over time, but they suffer from colour coding and clutter over time and multiple user trials. Hence, Blascheck et. al [15] utilizes hierarchical visualization approaches that take into account the inherent hierarchies in AoI clusters over time. Multiple visualization tools such as transition trees, graphs, transition matrix and hierarchy diagrams have been used to collectively portray the sequential, relational and temporally evolving nature of areas of interest in eye gaze data. A transition Tree based approach [68] combines scarf plot with an additional space-filling icicle plot diagram that provides a hierarchy of gaze transition sequences. But visual representations alone as described above can suffer from visual clutter, particularly when dealing with long duration data. While transition trees, graphs and scarf plots suffer from the same big data problem, transition matrices cannot convey the time evolving nature of the AoIs. In transition trees, for example, the arrows depicting transitions between AoIs can become cluttered over time. 



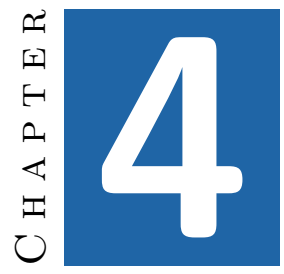

\section{Contributions}

Chapter 2 highlights the challenges in visual data analysis of large temporal data sets and briefly discussed about the different data abstraction and sequence analysis methods. Chapter 3 concentrates on the specific category of data simplification using symbolic approximation for 1D time-series data and the special category of $2 \mathrm{D}$ spatio-temporal data with spatio-temporal discontinuity and provides a brief introduction to the state of the art methods. This chapter summarizes every published articles and the contributions of the author of this thesis in every published article. The published articles can be categorized based on the dimensionality of the time-series data,

1. Data adaptive, efficient symbolic approximation of 1D time-series data with query-by-sketch pattern matching (Paper A).

2. Symbolic approximation of very large spatio-temporal data with spatio-temporal discontinuity (eye gaze data) and sequential analysis of symbolic data using Hidden Markov Model, Sequence mining (Paper B - Paper E).

\subsection{Summary of papers}

This section summarizes the aim, method, results and summary of contributions made by the author of this thesis for each appended publication. Please refer to the appended publications for detailed descriptions of the methods and application examples. 


\subsubsection{Paper A}

Simplification of the raw time-series data using symbolic approximation (for example, SAX [75]) is one of the most popular methods in time-series data mining. Once the raw data is converted into a symbol sequence, identification of repeating patterns and anomalies [107] can be performed through grammar based approaches as in Sequitur [89]. Since the grammar based methods are 'greedy' grammar induction algorithms, they are not always complete [106] and hence finding all possible patterns using a smallest complete grammar is an NP-hard problem [74]. One of the common solutions is to focus the search to identify only interesting patterns specified by a domain user [121]. In this thesis, Paper A presents an efficient shape grammar based approach for hierarchical, data adaptive symbolic approximation of long time-series data and interactive query-by-sketch based pattern identification.

\section{Aim}

A freehand sketch-based approach provides an interactive interface for domain users to define patterns of interest which can then be identified algorithmically in time-series data sets. Previous user query methods used the extensive process of (1) browsing through the entire data to find patterns of interest, (2) specify methods for selecting such patterns and search for them in the rest of the data. They included rigid constraints in query specification and pre-defined pattern templates for user query input. As this method is tiresome, there is a need for creating a visual interface to sketch any patterns of interest by the domain specialist. Since commonly the aim is to search for local trends in the time-series data, a symbolic approximation algorithm should preserve local information such as perceptually important points. Hence, the objectives of Paper A are listed as follows,

1. An interactive visual interface to sketch any patterns of interest by the domain specialist.

2. Data simplification using symbolic approximation that preserves the shape characteristics on a local scale.

3. A computationally efficient system for domain user interaction.

\section{Method}

While searching for user-sketched patterns, users often ignore differences across amplitude, scale and translation $[20,63,108]$. Any pattern search algorithm should be able to handle these three conditions in an efficient manner. The method discussed in the paper involves three basic steps,

Step 1: Shape grammar A visual grammar is extracted from the time-series by considering the data as a combination of basic elementary shapes positioned 


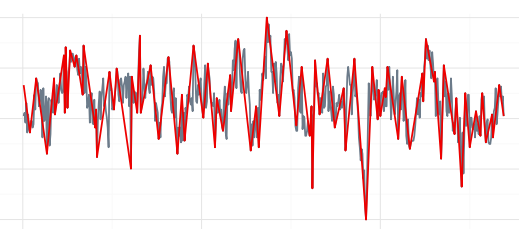

(a) Monthly values of the The Southern Oscillation Index - changes in air pressure related to sea surface temperatures in the central Pacific Ocean.

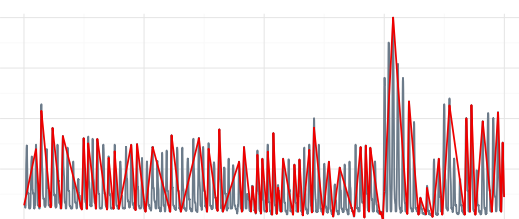

(b) A time-series showing a patients respiration (measured by thorax extension), as they wake up.

Figure 4.1: Locally adaptive data smoothing across different data sets. The red lines indicate the approximated input data. Significant anomalies are still retained in the approximation step.

across different amplitudes. These basic shapes are represented using a ratio and the ratios are used to transform the data into a symbolic representation.

Step 2: Hierarchical locally adaptive approximation. Since the aim is to search for local trends in the time-series data, insignificant spikes and valleys that would not alter the local trend can be approximated. Hence, the users are given the flexibility of performing local approximation of raw time-series data in a hierarchical manner. No extra arithmetic calculation is necessary since the entire approximation process is performed on the symbolic data using Regular expressions (RE). The advantage of performing a locally adaptive approximation rather than a piecewise adaptive approximation (PAA) is that the latter can potentially smooth out important points in the data set due to the constant sized bins used to compute the average of all the time points within that bin (see Figure 4.1).

Step 3: User-sketched pattern matching. Users can sketch approximate patterns in a separate sketching space, either through line strips defined through a sequence of mouse clicks or free form sketching. The user input is transformed into a symbolic representation using the above steps and a string matching, based on regular expressions (RE), is then performed in real-time to identify similar patterns. This symbolic search also permits the analyst to interactively carry out a step-wise relaxation of the constraints placed upon the search to find more sequences that match the general shape of the search sequence but less precisely. This relaxation requires only an editing of the grammar and no re-computation from the time-series and so can also be carried out in milliseconds, even for millions of time samples.

\section{Results}

The effectiveness of the method is demonstrated in a case study on stock market data although it is applicable to any numeric time-series data. The example data set was downloaded from the NYSE database for the ticker symbol ADM from 1st 


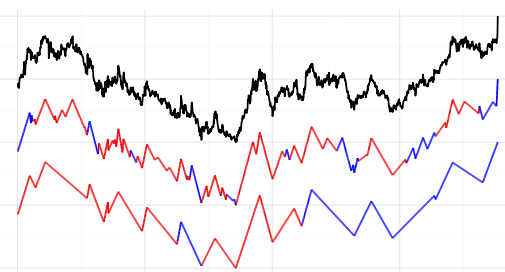

(a) Case 1: Incremental smoothing of the raw data and searching for head and shoulders pattern matches.

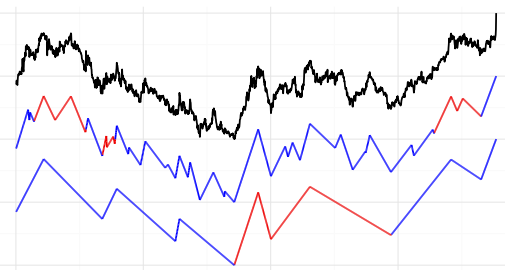

(b) Case 2: Incremental smoothing and searching of Double top pattern.

Figure 4.2: Hierarchical smoothing and pattern matching. The resulting matches are shown in red.

May 1997 to 30th October 2004. The input data consists of 1886 time points. One of the prime features of the algorithm is the ability to identify both short and long term patterns with soft constraints that are invariant across scale, amplitude and translation. The input will be in the form of rough user sketches and a RE based string matching is performed in real-time to search for matches. In Figure 4.2, the raw time-series data is displayed in black, the hierarchically approximated data at two different levels of approximation is displayed in blue. Two example patterns from financial domain are sketched in a separate sketching space and the matching patterns are displayed in red.

Case 1: 'Head and shoulders' pattern. This pattern in the financial domain is made up of a peak followed by a higher peak and then a lower peak. The displayed matches at two different levels of approximation is highlighted in red in the Figure 4.2(a).

Case 2: 'Double top'. Double tops or double bottoms in the financial domain consist of two peaks or troughs of similar magnitude as shown in the Figure 4.2(b). Due to the flexibility of the algorithm in providing for soft-constraints, two similar peaks are highlighted. At the same time, the algorithm does not allow for false matches that completely distort the trend in the pattern.

\section{Summary of contributions}

Based on the initial Query-by-sketch pattern matching idea of the co-authors, the author of this thesis performed a review of the existing literature to identify the complexities in the existing approaches and came up with an initial idea of the shape grammar approach for symbolic approximation, hierarchical locally adaptive approximation for smoothing the time-series and implemented the idea in a webbased user interface. The author of this thesis wrote an initial draft of the paper which was refined iteratively along with the co-authors. Query-by-sketch approach, 
shape grammar and use cases were refined and improved through discussions with the co-authors of this paper.

\subsubsection{Paper B}

Significant progress has been made recently in the data analysis and visualization of spatio-temporal geographic movement trajectories [8]. While there is a spatiotemporal continuity in the movements of physical objects, there are other spatiotemporal data sets such as eye gaze data that have the same structure such as position coordinates, time stamp, object id but with a significant difference. Eye movements include instantaneous jumps across the stimulus [6]. Hence it restricts the use of movement analysis methods for analyzing eye movement data since the spatio-temporal continuity are not the same for human or animal mobility and eye movements. This thesis focuses on this specific challenge of analyzing data sets with such spatio-temporal discontinuity for example in case of very long duration eye gaze data sets collected across multiple users.

\section{Aim}

Raw eye gaze data contains 2D spatial information of gaze coordinates over a stimulus along with the timestamp information. Eye gaze samples are labelled into fixations - periods of gaze over a region of the stimulus and saccades - movements between fixations as the viewer switches the attention. Fixation samples are labelled further using two prominent approaches - Area of Interest and Object of Interest (OoI) based methods. The distinction between two approaches lies in what questions they aim to answer. AoI based approaches reveal how a user inspects and monitors different regions of a scene while OoI based approaches reveal which distinct objects in the scene attract user attention. AoI based approaches can have a greater level of detail than OoI, as the total number of OoI labels is tied to the number of distinct objects in the scene. The transformation of raw gaze data into labels enables the use of sequence mining approaches to identify patterns of behaviour. The aim of this Paper B is to review the present state of the art on AoI labelling and the challenges in labelling AoIs in very long duration eye tracking data. For example, an eye tracker with a refresh rate of $60 \mathrm{~Hz}$, generates approximately 320000 samples of spatio-temporal data over a 90 minute recording session. Paper B proposes scope for improvement through the use of temporal clustering of fixation points to identify AoIs and convert the raw eye gaze data into a symbolic approximation where each symbol corresponds to a cluster label.

\section{Results}

The paper proposes research directions for a visual analysis approach based on using clustering and sequence analysis methods for analyzing the AoIs in long duration 
eye tracking data sets. The paper presented a two step approach: (1) to use fast clustering algorithms that can identify temporally evolving AoIs and (2) Interactive exploration of eye gaze patterns across AoIs using an interactive sequence mining approach - the ActiviTree method [117]. Using this approach, users can analyse how the transition between AoI sequences has evolved over time and identify AoI sequence paths followed by different users based on their significance determined by a data analyst.

\section{Summary of contributions}

The author of this thesis reviewed the existing literature to summarize the different methods for identification of AoIs and the challenges in data adaptive AoI identification in case of long duration eye tracking data sets. An initial draft version of the paper was prepared by the author of this thesis and iteratively improved along with the co-authors. The co-authors proposed the idea of using sequence mining to analyze the symbolically approximated eye gaze data.

\subsubsection{Paper C}

In long duration eye tracking studies, an eye tracker with a refresh rate of $60 \mathrm{~Hz}$ generates approximately 320000 samples of spatio-temporal data for a 90 minute recording session. Paper $\mathrm{C}$ presents an efficient data abstraction technique that transforms the raw data into a symbol sequence using clustering and hierarchical cluster merging suited for spatio-temporal eye tracking data sets with spatiotemporal discontinuity.

\section{Aim}

Clustering methods are not suitable for direct application on long-duration data sets as when the amount of eye gaze data increases, the local AoIs get distorted or saturated resulting in very few clusters (AoIs) that were accumulated over time. Hence, in order to analyze very long duration eye tracking data sets, the aim of Paper $\mathrm{C}$ include,

1. Data adaptive transformation of the raw eye gaze data into a symbolic representation without data saturation, where each symbol corresponds to an AoI cluster label.

2. A computationally efficient approach that can handle very long-duration eyetracking data.

3. A visualization system for interactive visual analysis providing a summary view of the identified AoIs over the entire duration of the data. 


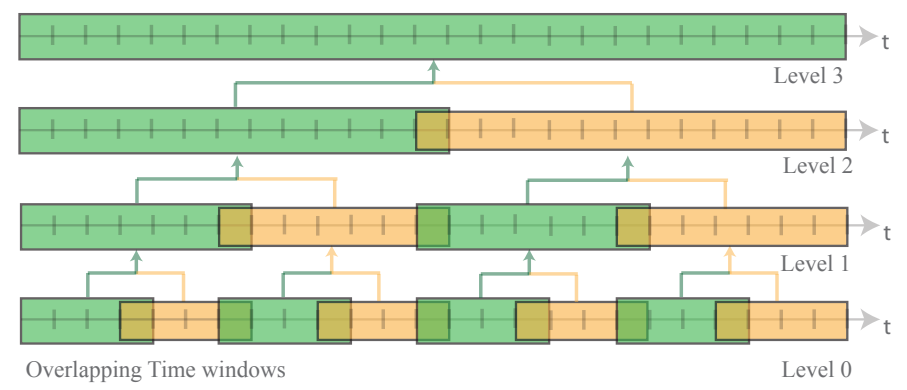

Figure 4.3: Hierarchical diagram of clustering and hierarchical cluster merging. At level 0 , the raw eye gaze data is divided into overlapping time windows and clustering is performed in each time window. Clusters from adjacent time windows are merged in a hierarchical manner at higher levels. Computations in a level are independent of each other and hence can be done in parallel.

\section{Method}

The raw eye gaze points are divided into overlapping time windows and a Minimum Spanning Tree (MST) based clustering algorithm [123] is used for clustering raw data points within each time window. A drawback with performing clustering over time windows is that a visual attention cluster (AoI) can spread across adjacent time windows. This would eventually lead to different cluster labels, as the clustering in a single time window is independent of the rest. This problem can be overcome by combining the cluster polygons from a set of neighbouring time windows using an MST based approach, where the similarity between two cluster polygons can be computed using a cost function. This merging process can be carried out in a hierarchical manner which creates a spatio-temporal hierarchy, as shown in Figure 4.3. One important advantage of this approach is that the computations in each level of the hierarchy can be parallelized and hence computationally efficient. The spatio-temporal hierarchy of levels shown in Figure 4.3 can be explored by the analyst to view the temporally evolving AoIs. The analyst can select a particular level of granularity from which assigned AoI labels (and colours) can be propagated downwards to shorter time windows.

The clustering results can be displayed in a space-time cube [43], as shown in Figure 4.4(b). The STC's horizontal plane corresponds to spatial coordinates, in this case spatial positions of eye gaze points, and the vertical axis corresponds to time going upward.

\section{Results}

Eye gaze data of two operational air traffic controllers (ATCOs) conducting a training session on a simulation of flights into a medium-sized airport was considered for the analysis. The training session lasted for around 85 minutes generating 

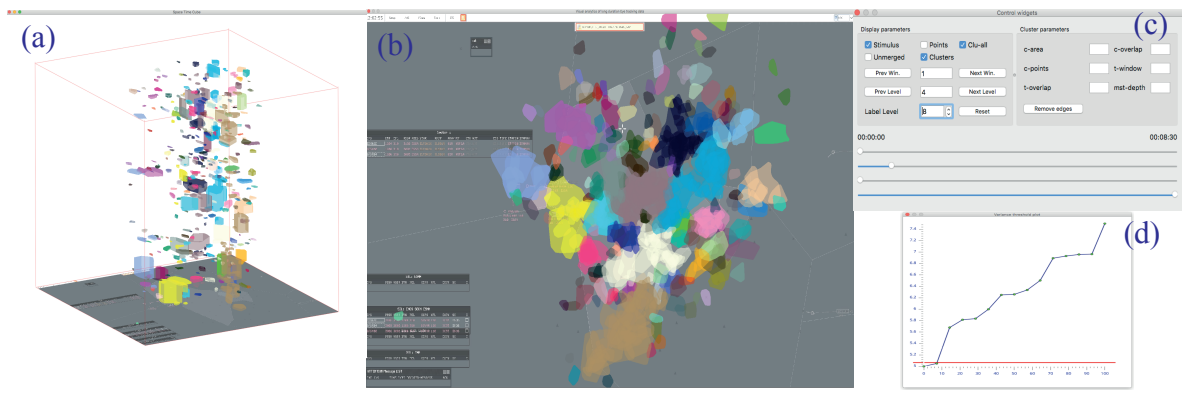

Figure 4.4: The main views of application interface: (a) Space-Time Cube displaying temporal aspects of the data and Aols, (b) main 2D visualization window showing the raw data and/or Aols, (c) interactive control panel, (d) interactive 2D plot for modifying clustering parameters.

approximately 320000 samples. The computation time for identifying AoIs in the example data sets was between 33 to 52 seconds for identification of AoIs. The results are displayed in a interactive visual analysis system as shown in Figure 4.4.

By browsing through the lower levels of the data hierarchy 4.3 for Air Traffic Controllers $\boldsymbol{A}$ and $\boldsymbol{B}$, the time windows at level 5 of the hierarchy were considered for analysis. As shown in Figure 4.5(a), (b) the two ATCOs show greater similarity in the first third of the scenario run-time, with comparable levels of sparsity. Based on the in-context visualization, it can be concluded that the AoIs correspond to the attention of ATCOs on aircraft moving along two routes, from the top and bottom left of the display towards the centre of the screen.

After that point, however, the STC (see Figure 4.5(g)) for ATCO- $\boldsymbol{A}$ remains similar in sparsity at the periphery but with greater focus on the airport (see Figure 4.5(e)). ATCO-B (see Figure 4.5(h)), however, begins to show greater focus on all parts of the airspace. One possible cause is that the number of aircraft has increased at this point and ATCO- $\boldsymbol{A}$ has continued to cope well with this increased traffic density, while ATCO- $\boldsymbol{B}$ is under increased pressure and may be coping less well. This may indicate different characteristics between the two ATCOs such as level of confidence, experience or, simply, quality of memory for details.

Data simplification through the AoI identification, together with the STC display, provides direct access to both spatial and temporal variation in the subject's attention. In summary, the hierarchical structure of merged AoIs in the system has allowed us to easily explore these long duration eye tracking data sets and identify the differing characteristics of ATCO- $\boldsymbol{A}$ and ATCO- $\boldsymbol{B}$. 


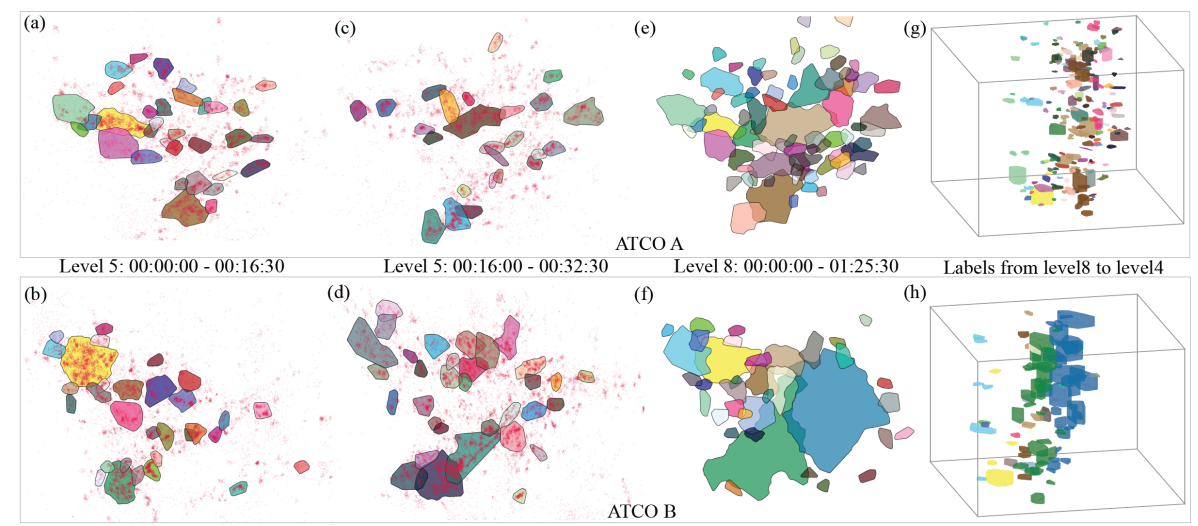

Figure 4.5: Identification of Aols for two ATCOs working on the same training scenario, lasting $\approx 85$ minutes. $(a)$ and $(b)$ show the Aols detected for the first $\approx 16$ minutes of the data for each ATCO at level 5 of the merged hierarchy, while (c) and (d) show the same result for the second $\approx 16$ minutes of the data. (e) and ( $f$ ) show the Aols identified in the entire data set at the highest level in the hierarchy and the difference in behaviour is clear in sub-figure ( $f$ ) where few, large Aols remain. The STC representations $(\mathrm{g})$ and $(\mathrm{h})$, with colours propagated down from level 8 to level 4 , highlights the differences in behaviour such as sparse and detailed attention.

\section{Summary of contributions}

The author of this thesis made a review of the existing literature to identify the disadvantages in clustering large eye gaze data sets and came up with the initial idea of using a parallelized hierarchical approach for merging cluster polygons using Minimum spanning trees. Along with the co-authors of the paper, extensive discussion were made to come up with a cost function for defining similarity between two cluster polygons. The author of the thesis also implemented the idea in a visual analysis application, prepared an initial draft of the paper with its accompanying images and identified the application case among the eye tracking data sets for demonstrating the effectiveness of the method.

\subsubsection{Paper D}

Eye tracking studies typically lasting for around 90 minutes are used to study the activities of Air traffic controllers (ATCO) and the analysis of their eye gaze movements can be used to improve the effectiveness of the training process.Paper $\mathrm{C}$ deals with the analysis of eye tracking data sets collected from different ATCOs working in a Remote tower environment. 


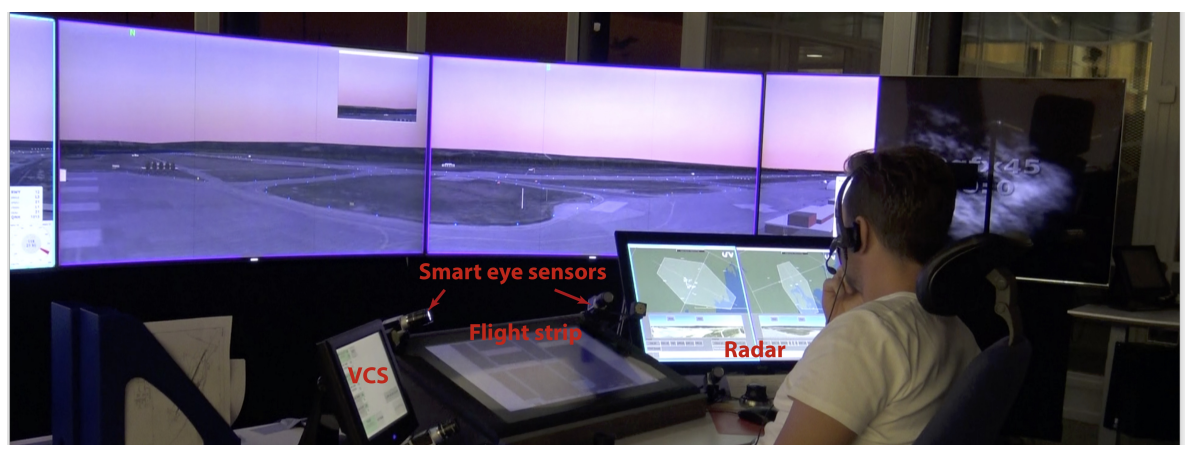

Figure 4.6: The experimental setup used in the case study consists of six screens making up the simulated tower view with three supporting screens placed on the desk. The six cameras of the SmartEye eye tracking system can be seen strategically positioned around the subject's workstation.

\section{$\operatorname{Aim}$}

The aim of this work is to analyze eye tracking data sets recorded from experienced air traffic controllers performing simulated training scenarios in a virtual remote tower environment. The data is analyzed along with domain experts and a professional ATCO where a number of practical examples were identified using methods and visualization tools described in Paper C.

\section{Method}

The experimental setup for the remote tower environment includes radar, flight strip, VCS (Voice Com System) systems and six large displays representing two remote airfields (see Figure 4.6). The SmartEye [111] gaze tracking system used in these experiments used six cameras, arranged around the controller, providing a wide field of view and so a comfortably large recording volume. The recorded eye gaze data is in the form of separate screen coordinates for each of the display screens at which the controller may be looking. The raw eye gaze data is preprocessed before analysis to create a single volume of data which includes the data from each of the individual screens as shown in Figure 4.8. The hierarchical clustering and cluster merging techniques described in Paper $\mathrm{C}$ are used to compute the areas of attention in the raw eye-tracking data.

\section{Results}

The dual remote tower scenario is predefined and incorporates a variety of events over time including the arrival of new approaching flights and scheduled departures, variations in visibility due to weather, and ground vehicle movements. Along with 


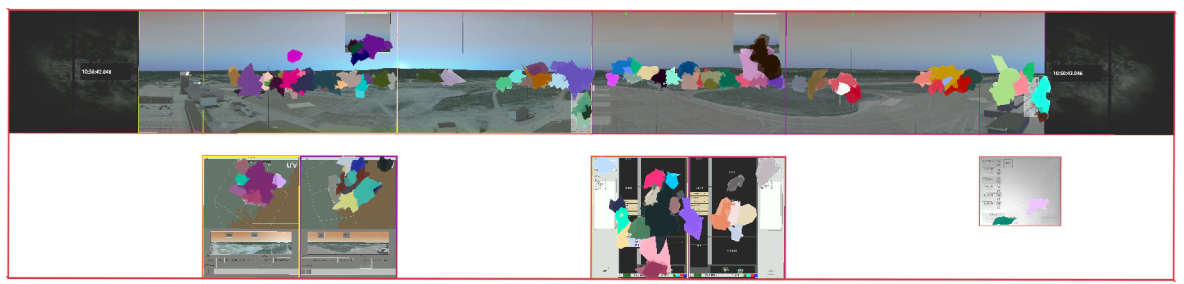

Figure 4.7: 2D 'top-down' view with AoAs. The two out-the-window (OTW) views arranged along the top. The two radar displays, the two strips displays and the VCS display are arranged along the bottom.
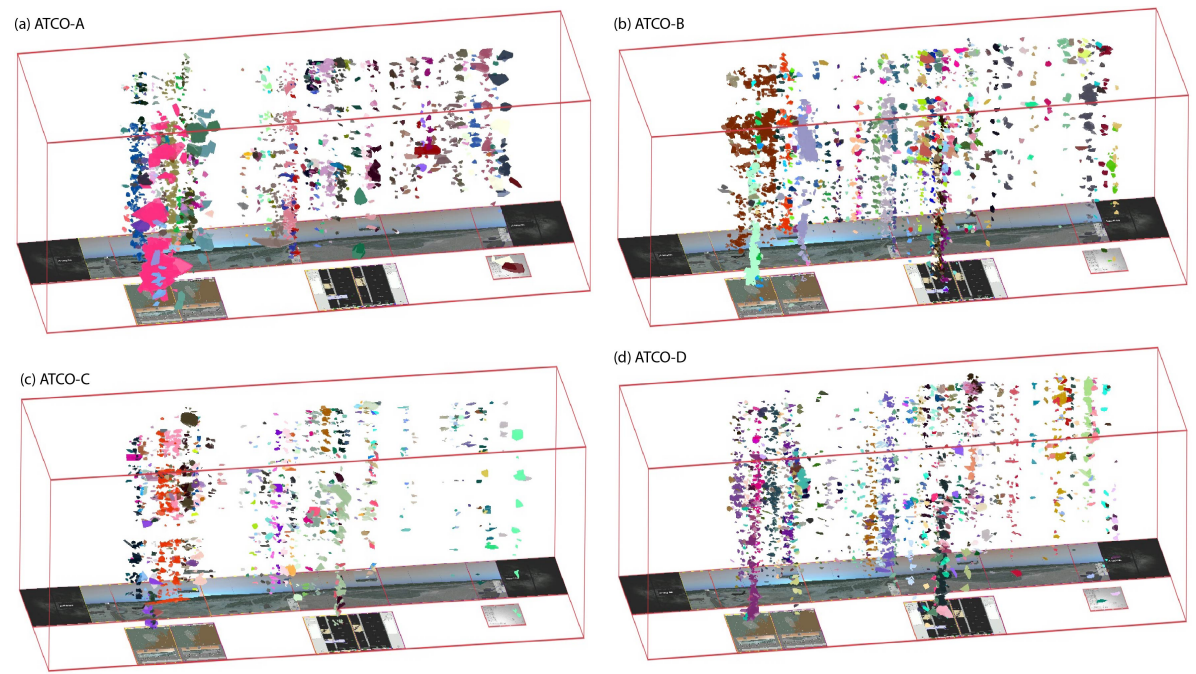

Figure 4.8: AoAs of four air traffic controllers carrying out the same scenario. The differences in visual attention such as sparse visual attention in case of ATCO-C can be observed in (c).

domain specialists and a professional ATCO, few scenarios of interest were identified and reviewed for corresponding analysis. A $2 \mathrm{D}$ visualization of the entire data from a single controller as shown in Figure 4.7 enables identification of patterns such as accumulation of visual attention at both ends of the runway and less visual attention in the middle of each runway. Comparison between different controllers allows the analyst to explore the variation in behaviour under similar circumstances. By comparing the four ATCOs as shown in Figure 4.8, we can identify patterns such as ATCO-C having a significantly more sparse attention than the others, ATCO-A clearly spending far more time focused upon the radar display. 

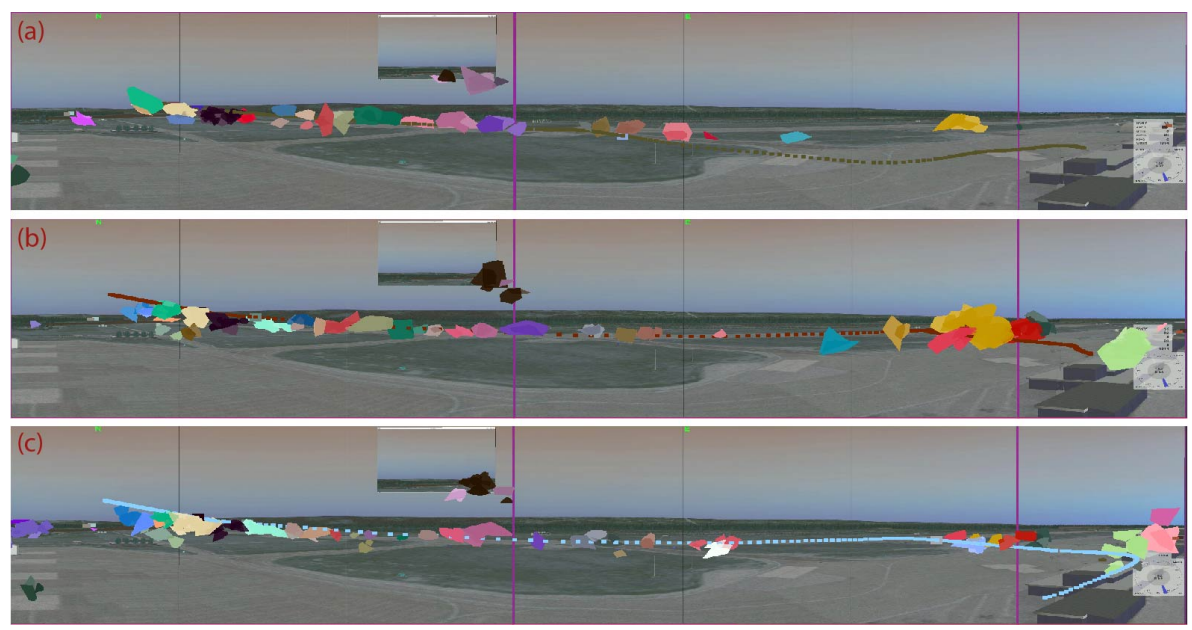

Figure 4.9: For an ATCO monitoring different landing simulations of aircraft, the similarity in AoAs of the ATCO can be identified in (a)-(c).
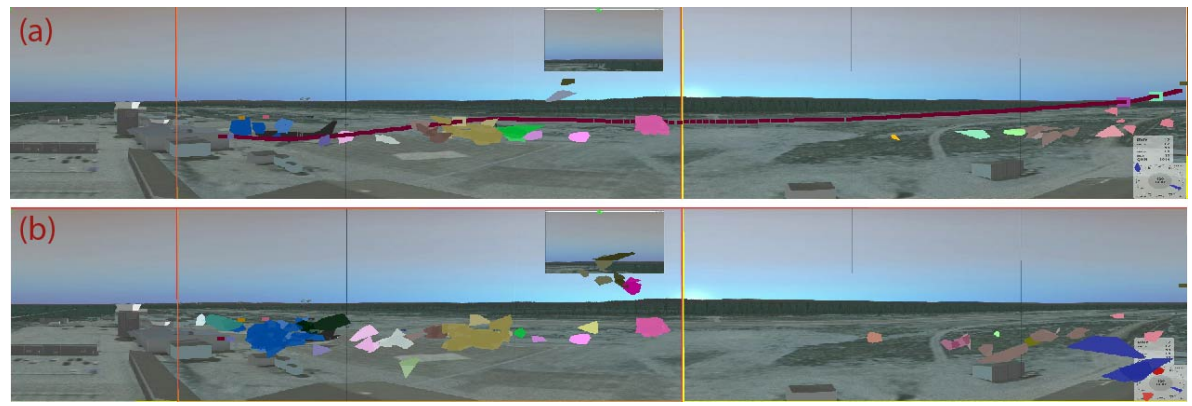

Figure 4.10: Similarities in the scanning behaviour of an ATCO during the presence and absence of aircraft on the screen can be identified in (a) and (b).

Activities of controllers during specific events such as landing events were also studied. For example, ATCO-D pays repeated visual attention at specific areas on the display and this is highly similar for different landing events as shown in Figure 4.9(a)-(c). Recurrence in scanning behaviour during the presence and absence of events in the airport was also identified with ATCO-A as shown in Figure 4.10 .

Based on the identified user behaviours, the proposed approach is a starting point to analyse and compare a number of long-duration eye gaze data from operational air traffic controllers. It will be of benefit in defining, demonstrating and checking adherence to 'best practice' during the training process. 


\section{Summary of contributions}

The author of this thesis along with the co-authors who are domain experts and a professional air traffic controller identified different use cases to explore interesting visual scanning patterns in the collected data sets. The author of this thesis implemented the visual analysis system, generated the images, contributed in writing the method description and identified certain use cases such as similarity of eye gaze during landing events and similarity in scanning behaviour in the presence and absence of events.

\subsubsection{Paper E}

In previous publications, data abstraction of raw eye gaze data into areas of interest based labelling acted as a first step in identifying visual scanning patterns in eye tracking data sets. But the comparison of visual scanning behaviour between multiple users performing the same experiment can be performed only through manual analysis. Also the complexity of tuning multiple parameters for identification of area of interest (AoI) using clustering and cluster merging increases with the number of users.

\section{Aim}

In order perform an algorithmic analysis of comparing user behaviour across multiple users, the aim of Paper E include:

1. Identification of common area of interest labels across multiple users with minimal user input parameters.

2. Analysis of user behaviour using multinomial Hidden Markov Model and Sequence mining based approaches to uncover interesting behaviour in the presence of noisy eye gaze data.

\section{Method}

The method extends the approach of Paper C to identify common AoIs labels across multiple users. A 3D Minimum spanning tree based clustering approach is constructed from the $2 \mathrm{D}$ eye gaze co-ordinates, timestamp and an extension of Maximum Standard Deviation Reduction (MSDR) algorithm of [124] were utilized to identify Area of Interest clusters. These improvements to the earlier approach remove the restrictions in tuning multiple parameters such as size of time window, MST neighbourhood depth and inconsistency factor. For merging the area of interest clusters from neighbouring time windows, a symmetric Jaccard distance metric is used as the cost function for defining similarity between AoIs. Using this approach only highly spatially similar AoIs are merged and so the entire computation can be performed with no user intervention. 


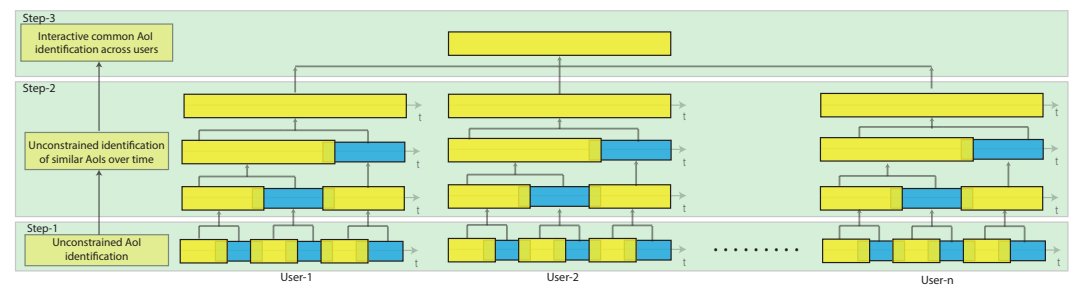

Figure 4.11: Method pipeline: Common Aol identification across user sets. Step 1: Identification of fixation clusters. Step 2: Similar Aol identification for every user. Step 3: Common Aol identification across all users.

In order to compute common AoI labels across all the subjects, an MST is computed for all the AoI clusters from the topmost time window of the hierarchy of each user as shown in Figure 4.11 and the merging process is repeated to compute similar AoIs from the MST. At this juncture, the analyst can also interactively modify a knee point plot to control the degree of similarity while merging the AoI clusters.

The common AoI labels from the topmost time window of the hierarchy (see Figure 4.11) can be propagated back to the lower levels containing original eye gaze points for each subject. The raw eye gaze data can be transformed into a symbolic representation where each eye gaze point can be labelled either as noise, saccade or AoI labels. Taking the noisy gaze data into account, two prominent sequence analysis approaches such as Hidden Markov Models (HMM) [13, 81, 99] and Sequence mining $[44,116]$ can be used to analyze behaviour across multiple subjects.

\section{Results}

The experimental setup from Paper D is used for collecting data from four operational air traffic controllers performing the same scenario. Each experimental scenario lasted for $\approx 90$ minutes for each controller. The common AoIs computed for four ATCOs carrying out the same scenario are displayed in Figure 4.12.

A multinomial HMM is used to analyze the AoI label sequence to identify common patterns across all the controllers. Figure 4.13 shows the most probable hidden state paths highlighting the similarities and differences of visual attention across different controllers. Using the Sequence mining tool — Eloquence [116], the order of visual attention between different areas of interest that are common across all the controllers can be investigated. For example, the "nested" back-and-forth scanning pattern across different AoIs after visiting the right airport radar (AoI label 3080, see Figure 4.14(b)) can be identified as shown in Figure 4.14(a).

By transforming the raw eye gaze data into symbolic representation where the symbol space is common across all users, sequence analysis methods such as 

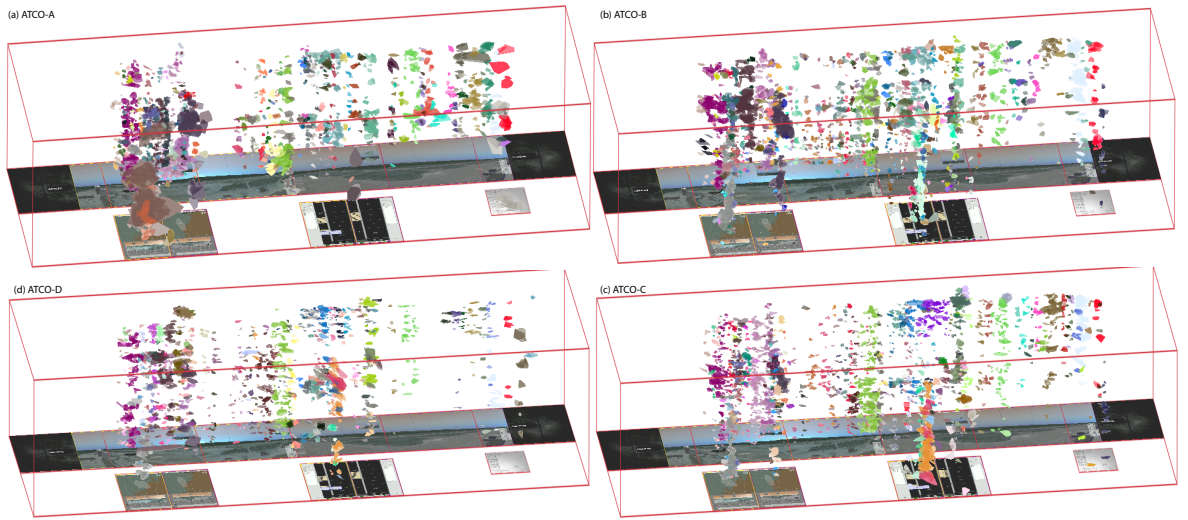

Figure 4.12: Common labelling of Aols across four controllers: Common Aols are marked with the same colour in each sub-figure. The Space-Time Cube (STC) can be rotated, zoomed and filtered along vertical axis to avoid visual clutter. The two tower views are displayed at the back of the cube while the radar, flight strip and the voice control displays are arranged across the front part of the cube.

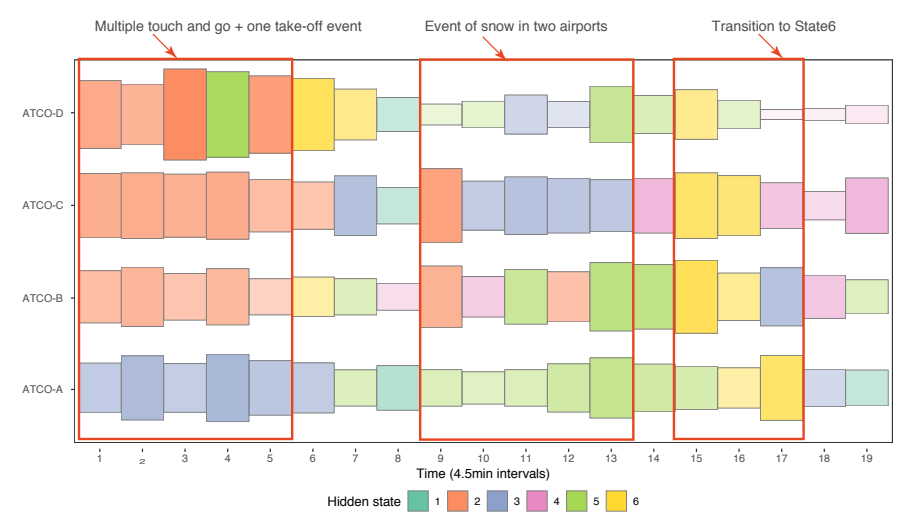

Figure 4.13: HMM state sequence for four controllers with information on contextual significance of each state given by colour intensity and size.

HMM and Sequence mining allows pattern identification and user driven analysis. While different sequence analysis methods from bio-informatics and text mining communities are available for analyzing the symbol sequence, two prominent approaches were used to demonstrate their usefulness for identifying patterns in large data sets. 


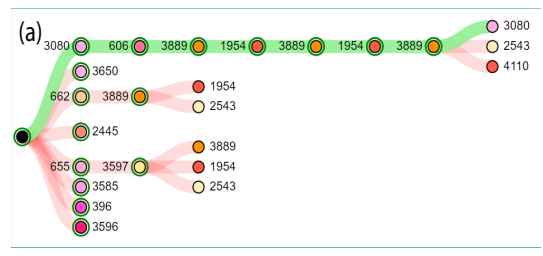

(a) Common eye-movements to all users.

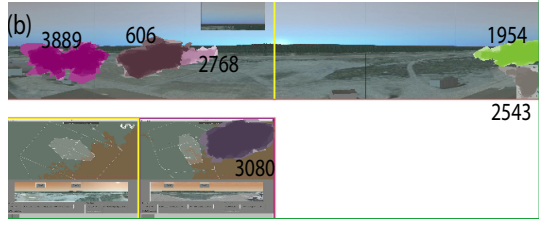

(b) Aols identified in the shown sequences.

Figure 4.14: Sequence analysis of common eye movements across all users Common eye-movements before and after visiting Aol 3080.

\section{Summary of contributions}

The author of this thesis proposed the idea of using different techniques for reducing the complexities of parameter tuning in identifying AoIs and computed the common AoI labels using an improved algorithm of Paper C. 


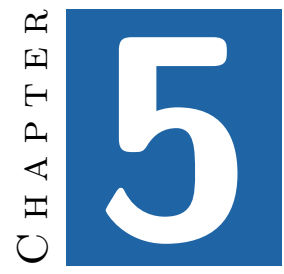

\section{Conclusion}

Visual analysis of large time-series data sets becomes complex due to large volumes of data that hinder efficient user interaction and pattern identification. In order to deal with the complexity this thesis focuses on different techniques for data abstraction by transforming the raw time-series data into symbolic approximations using efficient data processing algorithms. Sequence analysis is then used to identify patterns in the symbolic data set. This chapter describes how these goals have been met and to what degree.

\subsection{Data abstraction - Symbolic approximation}

When faced with large volumes of temporal data, transformation of raw data samples into symbolic approximations using data abstraction enables the use of different sequence analysis methods for data exploration which can also be user driven. But as discussed earlier, the dimensionality and characteristics of the temporal data dictates the choice of symbolic approximation methods.

In the case of $1 \mathrm{D}$ time series data, a shape grammar based approach was employed in Paper A to convert the raw time series data into a symbol sequence. The method provides a high degree of flexibility through user-defined and controlled data approximation. One of the advantages when compared with earlier methods is that the approximation retains perceptually important points without the need for storing additional information for each symbol in the sequence. Since the aim of the method was to search for patterns in the data set based on Query-by-Sketch, amplitude, scale and translation invariant pattern identification was necessary which was achieved through the shape grammar based approach. While using an 
intuitive sketch based interface for defining a query of interest, a domain analyst is required to provide only an approximate pattern or trend to define their input. This approach overcomes the difficulty of scanning through long time series to identify and select patterns of interest using different interaction techniques and then searching for related patterns across the entire data set. The technique operates completely in the symbol space for data smoothing and pattern identification, and hence is efficient for real-time data processing. While using symbolic approximation enables efficient data processing and data smoothing, it does not involve absolute value matching for pattern identification. For absolute value matching problems, further processing is required on the identified patterns of interest and the results can be filtered subsequently by comparing the symbolic data with the original time series data. The process of symbolic approximation also enables smoothing of time series data in a hierarchical manner, controlled by an analyst. It improves visual effectiveness by reducing visual clutter due to noise in long duration time series data.

In the case of $2 \mathrm{D}$ spatio-temporal data sets, the spatio-temporal continuity of the data samples determines the nature of algorithm to be used for symbolic approximation. Traditional data clustering algorithms used across geographic movement data sets such as human or animal migration cannot be applied directly to data sets with spatio-temporal discontinuity, for example, eye gaze data. Also, owing to the large size of 2D spatio-temporal data sets, identification of patterns over time suffers from data saturation. Hence, a clustering and hierarchical cluster merging algorithm described in Paper $\mathrm{C}$ was used for data abstraction. For example, Mean Shift clustering [21] was applied on a 4.5 minute time interval of a data set composed of 2D eye gaze points collected at $60 \mathrm{~Hz}$, as shown in Figures 5.1(a) and (b). In the example, high - (0.15) and low - quantile (0.01) values were used in Mean shift clustering, leading to clusters at either a very high or very low granularity. Clusters identified using the method described in Paper C, display more balanced results as shown in Figure 5.1(c). The spatio-temporal eye tracking data set was divided into overlapping time windows and the clusters from the adjacent time windows are computed by merging the clusters based on their strength of intersection and the merging process is repeated in a hierarchical manner.

The temporal eye gaze sequence is converted into a symbol sequence where each symbol corresponds to a cluster label. In order to compare the symbol sequence of multiple users, the identified cluster labels from one user should correlate with the labels from other users. Also, tuning of multiple parameters can increase the difficulty in scaling the method to multiple users. These difficulties were addressed in Paper E. In order to allow a user controlled data abstraction, the method allows tuning of a single parameter with the intuition that increasing or decreasing its value controls the degree of approximation. 


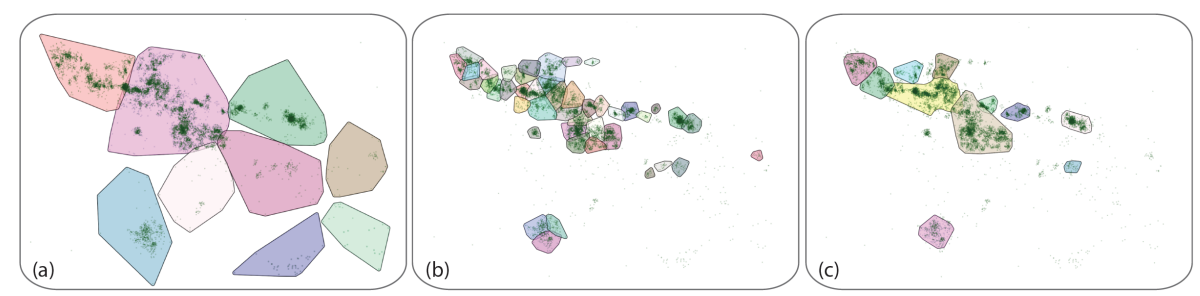

Figure 5.1: A comparison of clustering results on a 2D eye gaze data set collected at $60 \mathrm{~Hz}$ for a 4.5 minutes. Mean shift applied with (a) high (0.15) and (b) low quantile (0.01) values results in either too little and too much separation in the identified clusters. (c) Clusters identified using the method described in Paper C, display more balanced results.

\subsection{Sequence analysis of symbolic data}

Sequential analysis of symbol sequences can enable identification of patterns of interest from very large time-series data sets. The process of pattern search can be either user-driven or automatic depending on the needs of the users. Many grammarbased approaches can be used to compute repeating patterns and anomalies in the data. Since finding all possible patterns is an NP-hard problem, a domain user driven data exploratory visual analysis process that is interactive and efficient is an effective approach to gain information from the data. The important challenge lies in making a visual analysis process intuitive and interactive supplemented with effective visualizations that can portray the information with less cognitive overload. In the case of Paper A, the query-by-content method enables a usersketched pattern input and regular expression based pattern identification that is interactive and user-driven.

While the case of sequential data analysis of eye tracking data as described in Paper E, sequence mining of spatio-temporal eye tracking data is user-driven and interactive, the Hidden Markov model based sequence analysis, which is computationally expensive, can aid as a starting point for an exploratory data analysis process.

\subsection{Efficient data processing algorithms}

As we are dealing with long duration time series data sets, efficient data processing algorithms are necessary to enable an interactive user-driven and exploratory data analysis. In the case of 1D time series data, regular expression based algorithms were used for data abstraction and pattern search. Advanced regular expression string search algorithms as presented by Thompson [113] can increase the performance for millions of time series points. 
While analyzing 2D spatio-temporal eye tracking data, the hierarchical cluster merging process involves parallel computation across all time windows of a level in the hierarchy. Using such parallelization techniques enables efficient computation of symbol sequences from large temporal data sets.

\subsection{Future work}

During a query-by-sketch based approach for identifying patterns in 1D time series data, the matching patterns are scale, amplitude and translation invariant. As a future work, it would be interesting to rank the matches based on different similarity measures and hence compare the matched results. Also lower bounding constraints on symbolic approximation which is useful for efficient storage and retrieval is also an interesting option that can be pursued in the future [12].

In the case of $2 \mathrm{D}$ spatio-temporal data analysis, different evaluation techniques can be studied to compare the results of sequence analysis and identified patterns with the ground truth data that can be acquired from domain experts. Also, transformation of long duration eye tracking data into symbolic approximation opens up further possibilities for analyzing the data with different sequence analysis methods from text mining, bio-informatics and data mining communities. Additional information in eye gaze sequences such as underlying context of a eye gaze point can aid the pattern identification process with contextual information during sequential data analysis.

The methods developed for analyzing two dimensional eye tracking data sets opens up the possibilities of extending the ideas to dimensionality reduction methods dealing with high-dimensional time-series data sets. Methods such as timedependent dynamic t-SNE [100] achieves a trade-off between temporal coherence and projection reliability when reducing the high-dimensional data to lower dimensions. The spatio-temporal characteristics of the projected data over time can then be potentially analyzed using the methods developed in this thesis. 


\section{Bibliography}

[1] W. Aigner, S. Miksch, W. Müller, H. Schumann, and C. Tominski. Visual methods for analyzing time-oriented data. IEEE Transactions on Visualization and Computer Graphics, 14(1):47-60, 2007. [pages 2 and 15]

[2] W. Aigner, S. Miksch, W. Müller, H. Schumann, and C. Tominski. Visualizing time-oriented data - a systematic view. Computers $\mathscr{6}$ Graphics, 31(3):401409, 2007. [pages 4, 9, and 15]

[3] W. Aigner, S. Miksch, H. Schumann, and C. Tominski. Visualization of time-oriented data. Springer Science \& Business Media, 2011. [pages 1, 3, 4, 11 , and 15]

[4] M. Alam, K. M. Muttaqi, and D. Sutanto. A sax-based advanced computational tool for assessment of clustered rooftop solar pv impacts on lv and mv networks in smart grid. IEEE Transactions on Smart Grid, 4(1):577-585, 2013. [page 12]

[5] N. C. Anderson, F. Anderson, A. Kingstone, and W. F. Bischof. A comparison of scanpath comparison methods. Behavior research methods, 47(4):13771392, 2015. [page 24]

[6] G. Andrienko, N. Andrienko, M. Burch, and D. Weiskopf. Visual analytics methodology for eye movement studies. IEEE Transactions on Visualization and Computer Graphics, 18(12):2889-2898, 2012. [pages 23 and 31]

[7] G. Andrienko, N. Andrienko, C. Hurter, S. Rinzivillo, and S. Wrobel. Scalable analysis of movement data for extracting and exploring significant places. IEEE Transactions on Visualization and Computer Graphics, 19(7):10781094, 2012. [page 14]

[8] G. Andrienko, N. Andrienko, P. Bak, D. Keim, and S. Wrobel. Visual analytics of movement. Springer Science \& Business Media, 2013. [page 31]

[9] N. Andrienko and G. Andrienko. Exploratory analysis of spatial and temporal data: A systematic approach. Springer Science \& Business Media, 2006. [page 7] 
[10] N. Andrienko, G. Andrienko, G. Fuchs, S. Rinzivillo, and H.-D. Betz. Detection, tracking, and visualization of spatial event clusters for real time monitoring. In IEEE International Conference on Data Science and Advanced Analytics (DSAA), pages 1-10. IEEE, 2015. [page 10]

[11] B. Bach, P. Dragicevic, D. Archambault, C. Hurter, and S. Carpendale. A review of temporal data visualizations based on space-time cube operations. In Eurographics Conference on Visualization, 2014. [pages 11 and 25]

[12] R. Baeza-Yates and B. Ribeiro-Neto. Modern information retrieval, volume 463. ACM press New York, 1999. [page 46]

[13] L. E. Baum and T. Petrie. Statistical inference for probabilistic functions of finite state Markov chains. The Annals of Mathematical Statistics, 37(6): 1554-1563, 1966. [pages 13 and 40]

[14] T. Blascheck, K. Kurzhals, M. Raschke, M. Burch, D. Weiskopf, and T. Ertl. State-of-the-art of visualization for eye tracking data. In Proceedings of EuroVis, 2014. [page 20]

[15] T. Blascheck, K. Kurzhals, M. Raschke, S. Strohmaier, D. Weiskopf, and T. Ertl. AoI hierarchies for visual exploration of fixation sequences. In Proceedings of the Ninth Biennial ACM Symposium on Eye Tracking Research G6 Applications, pages 111-118, 2016. [page 25]

[16] P. Buono and A. L. Simeone. Interactive shape specification for pattern search in time series. In Proceedings of the Working Conference on Advanced visual interfaces, pages 480-481. ACM, 2008. [page 19]

[17] P. Buono, A. Aris, C. Plaisant, A. Khella, and B. Shneiderman. Interactive pattern search in time series. In Electronic Imaging 2005, pages 175-186. International Society for Optics and Photonics, 2005. [page 19]

[18] M. Burch, F. Beck, M. Raschke, T. Blascheck, and D. Weiskopf. A dynamic graph visualization perspective on eye movement data. In Proceedings of the Symposium on Eye Tracking Research and Applications, pages 151-158. ACM, 2014. [page 22]

[19] M. Card. Readings in information visualization: using vision to think. Morgan Kaufmann, 1999. [page 4]

[20] A. Chortaras and A. Schopenhauer. Efficient storage, retrieval and indexing of time series data. Master's thesis, Imperial College of Science, Technology and Medicine, 2002. [page 28] 
[21] D. Comaniciu and P. Meer. Mean shift: A robust approach toward feature space analysis. IEEE Transactions on pattern analysis and machine intelligence, 24(5):603-619, 2002. [page 44]

[22] A. Coutrot, J. H. Hsiao, and A. B. Chan. Scanpath modeling and classification with hidden markov models. Behavior research methods, 50(1):362-379, 2018. [page 24]

[23] F. Cristino, S. Mathôt, J. Theeuwes, and I. D. Gilchrist. Scanmatch: A novel method for comparing fixation sequences. Behavior research methods, 42(3): 692-700, 2010. [page 22]

[24] Q. Cui, M. Ward, E. Rundensteiner, and J. Yang. Measuring data abstraction quality in multiresolution visualizations. IEEE Transactions on Visualization and Computer Graphics, 12(5):709-716, 2006. [page 9]

[25] C. S. Daw, C. E. A. Finney, and E. R. Tracy. A review of symbolic analysis of experimental data. Review of Scientific instruments, 74(2):915-930, 2003. [page 18]

[26] M. F. De Oliveira and H. Levkowitz. From visual data exploration to visual data mining: A survey. IEEE Transactions on Visualization and Computer Graphics, 9(3):378-394, 2003. [pages 8 and 9]

[27] A. Dix and G. Ellis. By chance enhancing interaction with large data sets through statistical sampling. In Proceedings of the Working Conference on Advanced Visual Interfaces, pages 167-176. ACM, 2002. [page 8]

[28] G. Drusch, J. Bastien, and S. Paris. Analysing eye-tracking data: From scanpaths and heatmaps to the dynamic visualisation of areas of interest. Advances in Science, Technology, Higher Education and Society in the Conceptual Age: STHESCA, 20:205, 2014. [page 25]

[29] F. Du, B. Shneiderman, C. Plaisant, S. Malik, and A. Perer. Coping with volume and variety in temporal event sequences: Strategies for sharpening analytic focus. IEEE Transactions on Visualization and Computer Graphics, 23(6):1636-1649, 2016. [page 14]

[30] A. T. Duchowski, J. Driver, S. Jolaoso, W. Tan, B. N. Ramey, and A. Robbins. Scanpath comparison revisited. In Proceedings of the 2010 Symposium on Eye-Tracking Research 85 Applications, ETRA '10. ACM, 2010. [page 24]

[31] N. Elmqvist and J.-D. Fekete. Hierarchical aggregation for information visualization: Overview, techniques, and design guidelines. IEEE Transactions on Visualization and Computer Graphics, 16(3):439-454, 2009. [pages 8 and 9] 
[32] N. Elmqvist and P. Tsigas. A taxonomy of 3d occlusion management techniques. In 2007 IEEE Virtual Reality Conference, pages 51-58, 2007. [page 4]

[33] P. Esling and C. Agon. Time-series data mining. ACM Computing Surveys (CSUR), 45(1):12, 2012. [page 18]

[34] C. Faloutsos, M. Ranganathan, and Y. Manolopoulos. Fast subsequence matching in time-series databases. In Proceedings of the 1994 ACM SIGMOD International Conference on Management of Data, SIGMOD '94, pages 419429, 1994. [page 9]

[35] J.-D. Fekete. Visual analytics infrastructures: From data management to exploration. Computer, 46(7):22-29, 2013. [page 14]

[36] T. Fu. A review on time series data mining. Engineering Applications of Artificial Intelligence, 24(1):164-181, 2011. [page 18]

[37] M. M. Gaber, A. Zaslavsky, and S. Krishnaswamy. Mining data streams: a review. ACM Sigmod Record, 34(2):18-26, 2005. [page 18]

[38] J. H. Goldberg and J. I. Helfman. Scanpath clustering and aggregation. In Proceedings of the 2010 symposium on eye-tracking research 85 applications, pages 227-234. ACM, 2010. [page 22]

[39] M. Gregory and B. Shneiderman. Shape identification in temporal data sets. In Expanding the Frontiers of Visual Analytics and Visualization, pages 305-321. Springer, 2012. [page 19]

[40] Y. Gu, C. Wang, R. Bixler, and S. D'Mello. Etgraph: A graph-based approach for visual analytics of eye-tracking data. Computers 83 Graphics, 62:1-14, 2017. [page 23]

[41] D. Guo, M. Gahegan, A. M. MacEachren, and B. Zhou. Multivariate analysis and geovisualization with an integrated geographic knowledge discovery approach. Cartography and Geographic Information Science, 32(2):113-132, 2005. [page 9]

[42] D. Guo, J. Chen, A. M. MacEachren, and K. Liao. A visualization system for space-time and multivariate patterns (vis-stamp). IEEE Transactions on Visualization and Computer Graphics, 12(6):1461-1474, 2006. [page 7]

[43] T. Hägerstrand. What about people in regional science? Papers in regional science, 24(1):6-21, 1970. [page 33]

[44] J. Han, J. Pei, and X. Yan. Sequential pattern mining by pattern-growth: principle and extensions. Studies in Fuzziness and Soft Computing, 180: 183-220, 2005. [page 40] 
[45] M. C. Hao, M. Marwah, H. Janetzko, U. Dayal, D. A. Keim, D. Patnaik, N. Ramakrishnan, and R. K. Sharma. Visual exploration of frequent patterns in multivariate time series. Information Visualization, 11(1):71-83, 2012. [page 20]

[46] T. R. Hayes, A. A. Petrov, and P. B. Sederberg. A novel method for analyzing sequential eye movements reveals strategic influence on raven's advanced progressive matrices. Journal of Vision, 11(10):10-10, 2011. [page 24]

[47] H. Hochheiser and B. Shneiderman. Interactive exploration of time series data. In Discovery Science, pages 441-446. Springer, 2001. [page 18]

[48] H. Hochheiser and B. Shneiderman. Visual queries for finding patterns in time series data. University of Maryland, Computer Science Dept. Tech Report, CS-TR-4365, 2002. [page 18]

[49] H. Hochheiser and B. Shneiderman. Dynamic query tools for time series data sets: timebox widgets for interactive exploration. Information Visualization, $3(1): 1-18,2004$. [page 19]

[50] K. Holmqvist, M. Nyström, R. Andersson, R. Dewhurst, H. Jarodzka, and J. Van de Weijer. Eye tracking: A comprehensive guide to methods and measures. OUP Oxford, 2011. [page 20]

[51] C. Holz and S. Feiner. Relaxed selection techniques for querying time-series graphs. In Proceedings of the 22nd annual symposium on User interface software and technology, pages 213-222. ACM, 2009. [page 19]

[52] D. Jäckle, F. Fischer, T. Schreck, and D. A. Keim. Temporal mds plots for analysis of multivariate data. IEEE Transactions on Visualization and Computer Graphics, 22(1):141-150, 2015. [pages 3 and 15]

[53] A. K. Jain. Data clustering: 50 years beyond k-means. Pattern recognition letters, 31(8):651-666, 2010. [page 9]

[54] J. Johansson and C. Forsell. Evaluation of parallel coordinates: Overview, categorization and guidelines for future research. IEEE Transactions on Visualization and Computer Graphics, 22(1):579-588, 2015. [page 11]

[55] J. Johansson, P. Ljung, M. Jern, and M. Cooper. Revealing structure within clustered parallel coordinates displays. In IEEE Symposium on Information Visualization, pages 125-132, 2005. [page 9]

[56] C. Kanan, N. A. Ray, D. N. Bseiso, J. H. Hsiao, and G. W. Cottrell. Predicting an observer's task using multi-fixation pattern analysis. In Proceedings of the symposium on eye tracking research and applications, pages 287-290. ACM, 2014. [page 24] 
[57] S. Kandel, J. Heer, C. Plaisant, J. Kennedy, F. van Ham, N. H. Riche, C. Weaver, B. Lee, D. Brodbeck, and P. Buono. Research directions in data wrangling: Visualizations and transformations for usable and credible data. Information Visualization, 10(4):271-288, 2011. [page 15]

[58] D. Keim, G. Andrienko, J.-D. Fekete, C. Görg, J. Kohlhammer, and G. Melançon. Visual analytics: Definition, process, and challenges. In Information visualization, pages 154-175. Springer, 2008. [pages 7 and 14]

[59] D. A. Keim. Information visualization and visual data mining. IEEE Transactions on Visualization and Computer Graphics, 8(1):1-8, 2002. [page 10]

[60] D. A. Keim and H.-P. Kriegel. Visualization techniques for mining large databases: A comparison. IEEE Transactions on Knowledge and Data Engineering, 8(6):923-938, 1996. [page 8]

[61] D. A. Keim, C. Panse, M. Sips, and S. C. North. Visual data mining in large geospatial point sets. IEEE Computer Graphics and Applications, 24 (5):36-44, 2004. [page 9]

[62] E. Keogh, H. Hochheiser, and B. Shneiderman. An augmented visual query mechanism for finding patterns in time series data. In Flexible Query Answering Systems, pages 240-250. Springer, 2002. [page 19]

[63] E. J. Keogh and M. J. Pazzani. Relevance feedback retrieval of time series data. In Proceedings of the 22nd annual international conference on Research and development in Information Retrieval, pages 183-190. ACM, 1999. [page 28]

[64] T. C. Kübler, D. R. Bukenberger, J. Ungewiss, A. Wörner, C. Rothe, U. Schiefer, W. Rosenstiel, and E. Kasneci. Towards automated comparison of eye-tracking recordings in dynamic scenes. In 5th European Workshop on Visual Information Processing (EUVIP), pages 1-6. IEEE, 2014. [page 22]

[65] T. C. Kübler, E. Kasneci, and W. Rosenstiel. Subsmatch: Scanpath similarity in dynamic scenes based on subsequence frequencies. In Proceedings of the Symposium on Eye Tracking Research and Applications, pages 319-322, 2014. [page 22]

[66] N. Kumar, V. N. Lolla, E. J. Keogh, S. Lonardi, and C. A. Ratanamahatana. Time-series bitmaps: a practical visualization tool for working with large time series databases. In Proceedings of the 2005 SIAM International Conference on Data Mining, pages 531-535, 2005. [page 20]

[67] K. Kurzhals and D. Weiskopf. Space-time visual analytics of eye-tracking data for dynamic stimuli. IEEE Transactions on Visualization and Computer Graphics, 19(12):2129-2138, 2013. [page 25] 
[68] K. Kurzhals and D. Weiskopf. AoI transition trees. In Proceedings of the 41st Graphics Interface Conference, pages 41-48. Canadian Information Processing Society, 2015. [page 25]

[69] K. Kurzhals, F. Heimerl, and D. Weiskopf. ISeeCube: Visual analysis of gaze data for video. In Proceedings of the Symposium on Eye Tracking Research and Applications, pages 43-50. ACM, 2014. [page 25]

[70] K. Kurzhals, M. Hlawatsch, F. Heimerl, M. Burch, T. Ertl, and D. Weiskopf. Gaze stripes: Image-based visualization of eye tracking data. IEEE Transactions on Visualization and Computer Graphics, 22(1):1005-1014, 2016. [page 22]

[71] K. Kurzhals, M. Hlawatsch, C. Seeger, and D. Weiskopf. Visual analytics for mobile eye tracking. IEEE Transactions on Visualization and Computer Graphics, 23(1):301-310, 2016. [page 22]

[72] T. Lammarsch, W. Aigner, A. Bertone, M. Bögl, T. Gschwandtner, S. Miksch, and A. Rind. Interactive visual transformation for symbolic representation of time-oriented data. In International Workshop on Human-Computer Interaction and Knowledge Discovery in Complex, Unstructured, Big Data, pages 400-419. Springer, 2013. [page 17]

[73] H. Li and L. Yang. Time series visualization based on shape features. Knowledge-Based Systems, 41:43-53, 2013. [page 20]

[74] Y. Li and J. X. Chen. A nondeterministic approach to infer context free grammar from sequence. In Wavelet Active Media Technology and Information Processing, 11th International Computer Conference on, pages 1-9, 2014. [pages 12, 18, and 28]

[75] J. Lin, E. Keogh, S. Lonardi, and B. Chiu. A symbolic representation of time series, with implications for streaming algorithms. In Proceedings of the 8th ACM SIGMOD workshop on Research issues in data mining and knowledge discovery, pages 2-11. ACM, 2003. [pages 12, 18, 19, and 28]

[76] J. Lin, E. Keogh, S. Lonardi, J. P. Lankford, and D. M. Nystrom. Viztree: a tool for visually mining and monitoring massive time series databases. In Proceedings of the Thirtieth international conference on Very large data bases-Volume 30, pages 1269-1272. VLDB Endowment, 2004. [page 19]

[77] J. Lin, E. Keogh, L. Wei, and S. Lonardi. Experiencing sax: a novel symbolic representation of time series. Data Mining and knowledge discovery, 15(2): 107-144, 2007. [page 18] 
[78] J. Lin, S. Williamson, K. Borne, and D. DeBarr. Pattern recognition in time series. Advances in Machine Learning and Data Mining for Astronomy, 1: 617-645, 2012. [pages 3 and 18]

[79] L. Lins, J. T. Klosowski, and C. Scheidegger. Nanocubes for real-time exploration of spatiotemporal datasets. IEEE Transactions on Visualization and Computer Graphics, 19(12):2456-2465, 2013. [page 14]

[80] Z. Liu, B. Jiang, and J. Heer. immens: Real-time visual querying of big data. In Computer Graphics Forum, volume 32, pages 421-430. Wiley Online Library, 2013. [page 9]

[81] I. L. MacDonald and W. Zucchini. Hidden Markov and other models for discrete-valued time series, volume 110. CRC Press, 1997. [page 40]

[82] S. Miksch and W. Aigner. A matter of time: Applying a data-users-tasks design triangle to visual analytics of time-oriented data. Computers 86 Graphics, 38:286-290, 2014. [page 15]

[83] P. K. Mital, T. J. Smith, R. L. Hill, and J. M. Henderson. Clustering of gaze during dynamic scene viewing is predicted by motion. Cognitive Computation, 3(1):5-24, 2011. [page 23]

[84] P. K. Muthumanickam, C. Forsell, K. Vrotsou, J. Johansson, and M. Cooper. Supporting exploration of eye tracking data: Identifying changing behaviour over long durations. BELIV '16, pages 70-77. ACM, 2016.

[85] P. K. Muthumanickam, K. Vrotsou, M. Cooper, and J. Johansson. Shape grammar extraction for efficient query-by-sketch pattern matching in long time series. In IEEE Conference on Visual Analytics Science and Technology (VAST), pages 121-130, 2016. [page 18]

[86] P. K. Muthumanickam, J. Helske, A. Nordman, J. Johansson, and M. Cooper. Comparison of attention behaviour across user sets through automatic identification of common areas of interest. In Proceedings of Hawaii International Conference on System Sciences, HICSS, 2019.

[87] P. K. Muthumanickam, A. Nordman, L. Meyer, S. Boonsong, J. Lundberg, and M. Cooper. Analysis of long duration eye-tracking experiments in a remote tower environment. In 13th USA/Europe air traffic management RED seminar, 2019.

[88] P. K. Muthumanickam, K. Vrotsou, A. Nordman, J. Johansson, and M. Cooper. Identification of temporally varying areas of interest in longduration eye-tracking data sets. IEEE Transactions on Visualization and Computer Graphics, 25(1):87-97, 2019. 
[89] C. G. Nevill-Manning and I. H. Witten. Identifying hierarchical structure in sequences: A linear-time algorithm. Journal of Artificial Intelligence Research, 7:67-82, 1997. [pages 12, 18, and 28]

[90] D. Noton and L. Stark. Scanpaths in eye movements during pattern perception. Science, 171(3968):308-311, 1971. [page 24]

[91] E. A. Over, I. T. Hooge, and C. J. Erkelens. A quantitative measure for the uniformity of fixation density: The voronoi method. Behavior research methods, 38(2):251-261, 2006. [page 22]

[92] L. Paletta, K. Santner, G. Fritz, A. Hofmann, G. Lodron, G. Thallinger, and H. Mayer. A computer vision system for attention mapping in slam based 3d models. arXiv preprint arXiv:1305.1163, 2013. [page 22]

[93] A. Panuccio, M. Bicego, and V. Murino. A hidden markov model-based approach to sequential data clustering. In Joint IAPR International Workshops on Statistical Techniques in Pattern Recognition (SPR) and Structural and Syntactic Pattern Recognition (SSPR), pages 734-743. Springer, 2002. [page 13]

[94] Y. Park, M. Cafarella, and B. Mozafari. Visualization-aware sampling for very large databases. In International Conference on Data Engineering (ICDE), pages 755-766. IEEE, 2016. [pages 8 and 9]

[95] J. Pei, J. Han, B. Mortazavi-asl, J. Wang, H. Pinto, Q. Chen, U. Dayal, and M.-C. Hsu. Mining Sequential Patterns by Pattern-Growth : The PrefixSpan Approach. IEEE Trans on Knowledge and Data Engineering, 16 (11):1424-1440, 2004. [page 13]

[96] J. Pei, J. Han, and W. Wang. Constraint-based sequential pattern mining: the pattern-growth methods. Journal of Intelligent Information Systems, 28 (2):133-160, 2007. [page 13]

[97] H. Piringer, C. Tominski, P. Muigg, and W. Berger. A multi-threading architecture to support interactive visual exploration. IEEE Transactions on Visualization and Computer Graphics, 15(6):1113-1120, 2009. [page 14]

[98] C. M. Privitera and L. W. Stark. Algorithms for defining visual regionsof-interest: Comparison with eye fixations. IEEE Transactions on Pattern Analysis and Machine Intelligence, 22(9):970-982, 2000. [pages 22 and 24]

[99] L. Rabiner. A tutorial on hidden Markov models and selected applications in speech recognition. Proceedings of the IEEE, 77(2):257-286, 1989. [page 40] 
[100] P. E. Rauber, A. X. Falcão, and A. C. Telea. Visualizing time-dependent data using dynamic t-sne. In EuroVis (Short Papers), pages 73-77, 2016. [pages 3, 15, and 46]

[101] S. M. Ross et al. Stochastic processes, volume 2. John Wiley \& Sons New York, 1996. [page 24]

[102] K. Ryall, N. Lesh, T. Lanning, D. Leigh, H. Miyashita, and S. Makino. Querylines: Approximate query for visual browsing. In CHI'05 Extended Abstracts on Human Factors in Computing Systems, pages 1765-1768. ACM, 2005. [page 19]

[103] A. Santella and D. DeCarlo. Robust clustering of eye movement recordings for quantification of visual interest. In Proceedings of the 2004 symposium on Eye tracking research 6 applications, pages 27-34, 2004. [page 23]

[104] T. Santini, W. Fuhl, T. Kübler, and E. Kasneci. Bayesian identification of fixations, saccades, and smooth pursuits. In Proceedings of the Ninth Biennial ACM Symposium on Eye Tracking Research \& Applications, pages 163-170. ACM, 2016. [page 20]

[105] Y. Sawahata, R. Khosla, K. Komine, N. Hiruma, T. Itou, S. Watanabe, Y. Suzuki, Y. Hara, and N. Issiki. Determining comprehension and quality of tv programs using eye-gaze tracking. Pattern Recognition, 41(5):1610-1626, 2008. [page 23]

[106] P. Senin, J. Lin, X. Wang, T. Oates, S. Gandhi, A. P. Boedihardjo, C. Chen, S. Frankenstein, and M. Lerner. Grammarviz 2.0: a tool for grammar-based pattern discovery in time series. In Joint European Conference on Machine Learning and Knowledge Discovery in Databases, pages 468-472. Springer, 2014. [page 28]

[107] P. Senin, J. Lin, X. Wang, T. Oates, S. Gandhi, A. P. Boedihardjo, C. Chen, and S. Frankenstein. Time series anomaly discovery with grammar-based compression. In Proceedings of the 18th International Conference on Extending Database Technology, EDBT, pages 481-492, 2015. [pages 12 and 28]

[108] H. Shatkay and S. B. Zdonik. Approximate queries and representations for large data sequences. In Proceedings of the 12th International Conference on Data Engineering., pages 536-545, 1996. [page 28]

[109] B. Shneiderman. The eyes have it: A task by data type taxonomy for information visualizations. In The craft of information visualization, pages 364-371. Elsevier, 2003. [pages 4 and 7] 
[110] G. Shurkhovetskyy, N. Andrienko, G. Andrienko, and G. Fuchs. Data abstraction for visualizing large time series. In Computer Graphics Forum, volume 37, pages 125-144. Wiley Online Library, 2018. [pages 8, 9, 10, 15, and 18$]$

[111] Smart Eye AB, Gothenburg, Sweden. Smart Eye Pro. [Online]. Available: https://smarteye.se/. [page 36]

[112] N. H. Thach and E. Suzuki. A symbolic representation for trajectory data. The Japanese Society Artificial Intelligence, 1A2-2, 2010. [page 17]

[113] K. Thompson. Programming techniques: Regular expression search algorithm. Communications of the ACM, 11(6):419-422, 1968. [page 45]

[114] C. Tominski. Event-based concepts for user-driven visualization. Information Visualization, 10(1):65-81, 2011. [page 11]

[115] L. vd Maaten, E. Postma, and H. vd Herik. Dimensionality reduction: A comparative review. Technical Report, Tiburg Centre for Creative Computing, Tilburg University (TR 2009-005), 2009. [page 9]

[116] K. Vrotsou and A. Nordman. Exploratory visual sequence mining based on pattern-growth. IEEE Transactions on Visualization and Computer Graphics, 25(8):2597-2610, 2019. [pages 24 and 40]

[117] K. Vrotsou, J. Johansson, and M. Cooper. Activitree: Interactive visual exploration of sequences in event-based data using graph similarity. IEEE Transactions on Visualization and Computer Graphics, 15(6):945-952, 2009. [pages 3, 13, and 32]

[118] K. Vrotsou, H. Janetzko, C. Navarra, G. Fuchs, D. Spretke, F. Mansmann, N. Andrienko, and G. Andrienko. Simplifly: A methodology for simplification and thematic enhancement of trajectories. IEEE Transactions on Visualization and Computer Graphics, 21(1):107-121, 2014. [page 8]

[119] Z. Wang, N. Ferreira, Y. Wei, A. S. Bhaskar, and C. Scheidegger. Gaussian cubes: Real-time modeling for visual exploration of large multidimensional datasets. IEEE Transactions on Visualization and Computer Graphics, 23 (1):681-690, 2016. [page 14]

[120] M. Ward. Finding needles in large-scale multivariate data haystacks. IEEE Computer Graphics and Applications, 24(5):16-19, 2004. [page 9]

[121] M. Wattenberg. Sketching a graph to query a time-series database. In CHI'01 Extended Abstracts on Human factors in Computing Systems, pages 381-382. ACM, 2001. [pages 19 and 28] 
58 Bibliography

[122] M. Wattenberg. Arc diagrams: Visualizing structure in strings. In IEEE Symposium on Information Visualization, pages 110-116. IEEE, 2002. [page 20]

[123] C. T. Zahn. Graph-theoretical methods for detecting and describing gestalt clusters. IEEE Transactions on Computers, 100(1):68-86, 1971. [page 33]

[124] Y. Zhou, O. Grygorash, and T. F. Hain. Clustering with minimum spanning trees. International Journal on Artificial Intelligence Tools, 20(01):139-177, 2011. [page 39] 


\section{Papers}

The papers associated with this thesis have been removed for copyright reasons. For more details about these see:

http://urn.kb.se/resolve?urn=urn:nbn:se:liu:diva-162220 


\section{DEPARTMENT OF SCIENCE AND TECHNOLOGY}

Linköping Studies in Science and Technology. Dissertation No. 2030

Information Visualization

Division of Media and Information Technology

Department of Science and Technology (ITN)

Linköping University

SE-581 83 Linköping, Sweden

www.liu.se 